Cahiers de géographie du Québec

\title{
Analyse morphologique, classification et protection des paysages : le cas de Charlevoix
}

\section{Jean Raveneau}

Volume 21, numéro 53-54, 1977

URI : https://id.erudit.org/iderudit/021361ar

DOI : https://doi.org/10.7202/021361ar

\section{Aller au sommaire du numéro}

Éditeur(s)

Département de géographie de l'Université Laval

\section{ISSN}

0007-9766 (imprimé)

1708-8968 (numérique)

\section{Découvrir la revue}

\section{Citer cet article}

Raveneau, J. (1977). Analyse morphologique, classification et protection des paysages : le cas de Charlevoix. Cahiers de géographie du Québec, 21(53-54), 135-186. https://doi.org/10.7202/021361ar
Résumé de l'article

L'objectif de cette recherche est de trouver des méthodes d'analyse et de classification des paysages devant permettre l'application des articles de la Loi sur les biens culturels (Loi 2, 1972, Province de Québec) concernant la déclaration « d'arrondissements naturels ». La méthodologie proposée a été appliquée à la région de Charlevoix située à une centaine de $\mathrm{km}$ au nord-est de la ville de Québec.

On montre d'abord que les " arrondissements naturels " décrits dans la loi devraient plutôt porter le nom « d'arrondissements culturels ». L'identification des portions de territoire pouvant être déclarés arrondissements naturels ou culturels implique une analyse préalable des paysages de l'ensemble d'une région. Trois dimensions doivent être considérées : les formes, le peuplement, la perception. Dans cet article on aborde uniquement le problème de l'étude des formes des paysages, c'est-à-dire l'analyse morphologique. Quelques méthodes modernes d'analyse des paysages sont passées en, revue. Elles comportent généralement trois phases : inventaire, classification et évaluation proprement dite en fonction de critères particuliers (récréation notamment). La région de Charlevoix se prête particulièrement bien à l'étude des paysages en raison de sa topographie contrastée, de l'ancienneté de son peuplement, de l'empreinte d'activités économiques diversifiées. On a d'abord procédé à une analyse visuelle comportant le relevé des limites visuelles, la classification des points de vue et le relevé des types d'itinéraires routiers. On a ensuite dressé un inventaire spatial et monté une matrice géographique d'informations comportant 70 variables relevées sur la base de 47 zones de travail. On a choisi des variables qui structurent l'image des paysages et qui en conditionnent l'évolution, à la fois dans l'espace et dans le temps. La matrice a été traitée simultanément selon des méthodes visuelles (méthode Bertin) et quantitatives (analyse factorielle). Ce traitement a fait ressortir l'influence structurante du relief, de l'ancienneté et de la densité du peuplement, de l'importance du patrimoine historique. Quatre grands ensembles géographiques ont ainsi été dégagés, chacun comportant plusieurs sous-ensembles : les zones montagneuses sans population, les zones de peuplement récent du plateau, les vallées et leurs rebords, le littoral. Une seconde classification porte spécifiquement sur les variables pouvant permettre de définir le " potentiel culturel ». Elle conduit à une hiérarchisation des zones de Charlevoix en fonction de leur intérêt plus ou moins grand pour une désignation éventuelle comme arrondissement « naturel » (ou culturel). Cette classification est complétée par l'identification des diverses formes de pollution des paysages. La principale menace de dégradation des paysages provient des poussées d'urbanisation anarchique à la périphérie de Baie-Saint-Paul et La Malbaie.

En conclusion il apparaît que la notion même d'arrondissement culturel ne permet pas de répondre aux impératifs de protection et de mise en valeur des paysages. Il faut plutôt penser en termes de développement culturel global impliquant des actions d'intensité différente selon les types de paysages et les dangers de dégradation les affectant.
Ce document est protégé par la loi sur le droit d'auteur. L’utilisation des services d’Érudit (y compris la reproduction) est assujettie à sa politique d'utilisation que vous pouvez consulter en ligne.

https://apropos.erudit.org/fr/usagers/politique-dutilisation/ 


\title{
ANALYSE MORPHOLOGIQUE, CLASSIFICATION ET PROTECTION DES PAYSAGES : LE CAS DE CHARLEVOIX
}

\author{
par \\ Jean RAVENEAU \\ Département de Géographie, université Laval, Québec G1K 7P4
}

\section{ARRONDISSEMENTS NATURELS OU ARRONDISSEMENTS CULTURELS ?}

Partir d'un texte de loi pour établir un plan de recherche est une situation à laquelle les géographes sont et seront de plus en plus confrontés, même si la démarche s'avère peu conforme aux schémas conventionnels de la recherche scientifique. C'est ce type de défi que nous avons dû relever lorsque, en 1974, la Direction générale du patrimoine du ministère des Affaires culturelles du Québec (MAC) a demandé à un groupe de chercheurs du département de Géographie de l'université Laval d'élaborer une " méthodologie d'inventaire des sites et arrondissements naturels " et d'appliquer cette méthodologie à la région de Charlevoix ${ }^{1}$.

Le point de départ de la recherche émanait de l'article 45 de la Loi 2 , dite "Loi sur les biens culturels » ${ }^{2}$. Cet article s'énonce comme suit :

\begin{abstract}
"Le lieutenant-gouverneur en conseil peut, sur la recommandation du ministre qui prend avis de la Commission, déclarer arrondissement historique un territoire, une municipalité ou une partie d'une municipalité en raison de la concentration de monuments ou de sites historiques qui s'y trouvent. II peut également, de la même façon, déclarer arrondissement naturel un territoire, une municipalité ou une partie d'une municipalité en raison de l'intérêt esthétique, légendaire ou pittoresque que présente son harmonie naturelle "3.
\end{abstract}

Partant du texte de la Loi 2 il s'agissait d'établir une méthodologie permettant l'application de l'article 45 . Or, le moins qu'on puisse dire, est que l'énoncé de cet article est remarquablement ambigu, vague et imprécis dans sa formulation et son vocabulaire. Considérons tout d'abord le terme " arrondissement naturel»: il semble qu'il ait été utilisé par le législateur en opposition avec le terme "arrondissement historique ». Si l'on réfère à la définition littérale du dictionnaire, l'adjectif naturel désigne ce qui est "propre au monde physique, à l'exception de I'homme et de ses ceuvres ..." (Petit Robert). Or, de nos jours, bien peu de portions de l'espace terrestre n'ont pas été touchées par l'homme. De plus, peut-on logiquement parler de "l'intérêt légendaire " d'un arrondissement naturel sans une présence, actuelle ou passée, de l'homme dans ce lieu ? Enfin, sur le plan strictement politique, on peut contester la compétence du ministère des Affaires culturelles, chargé d'appliquer la Loi sur les biens culturels, à désigner et 
administrer des arrondissements naturels alors qu'il existe d'autres lois administrées par d'autres ministères, ayant des objectifs précis dans ce domaine. On peut citer par exemple les Lois sur les parcs provinciaux et la Loi sur les réserves écologiques appliquées respectivement par le ministère du Tourisme, de la Chasse et de la Pêche et le ministère des Terres et Forêts.

Pour toutes ces raisons, la distinction entre " arrondissement naturel» et "arrondissement historique " nous est apparue artificielle puisqu'elle introduit une séparation dans un continuum (l'espace terrestre) affecté à divers degrés par l'intervention de l'homme. La marque de l'homme, en termes d'utilisation du sol, de cadastre, d'éléments architecturaux, d'événements historiques, de traditions culturelles, etc., se superpose à un espace physique défini par le relief, la végétation, I'hydrographie, etc. Les deux ensembles sont indissociables l'un de l'autre et l'on ne peut pas abstraire les éléments historiques du cadre naturel qui les entoure. Le tout constitue un paysage que l'on peut qualifier de «culturel » en ce sens qu'il témoigne des caractères culturels de la population qui occupe le milieu. Certains de ces paysages présentent des traits distinctifs qu'il convient de protéger et de transmettre aux générations futures comme témoignages du patrimoine culturel d'un groupe humain déterminé. Dans l'esprit de la Loi sur les biens culturels, ces paysages pourraient être désignés comme arrondissements culturels dont certains seraient marqués intensément par la présence de I'homme alors que d'autres seraient dominés par les éléments naturels avec une présence humaine plus discrète. Ce changement d'appellation nécessiterait évidemment des amendements au texte de la Loi. Une fois définie la notion d'arrondissements culturel, il reste à déterminer des critères qui permettraient de formuler des recommandations en vue d'une désignation comme arrondissement de certaines parties d'un territoire. Sur quoi peut-on s'appuyer pour choisir ces critères?

\section{SUR QUELLES BASES ÉTABLIR DES CRITĖRES DE DÉTERMINATION D'ARRONDISSEMENTS CULTURELS ?}

Le texte de la Loi 2 dit que le lieutenant-gouverneur en conseil peut déclarer "arrondissement naturel " un territoire, une municipalité ou une partie d'une municipalité en raison de "l'intérêt esthétique, légendaire ou pittoresque que présente son harmonie naturelle ". II s'agit là de notions très subjectives, intuitives et difficilement quantifiables. La mise en œuvre de la Loi implique que l'on porte des jugements de valeur sur les paysages de différentes régions afin de déterminer ceux qui devraient être protégés. Une telle évaluation ne peut se concevoir sans une analyse préalable du contenu des paysages indépendamment de tout jugement de valeur. Chaque paysage humanisé est en lui-même un fait culturel résultant de l'action de l'homme sur le milieu. L'analyse qui en découle doit donc faire appel à trois dimensions distinctes mais étroitement liées entre elles:

- les formes

- le peuplement

- la perception 
D'une manière générale on peut dire que les formes des paysages (révélées par l'analyse morphologique) dans un espace donné sont la résultante de l'interaction entre les agents humains (révélés par l'analyse du peuplement) et le cadre physique qui les supporte, ceci à travers des systèmes de valeurs (révélés par l'analyse perceptuelle) conditionnant l'action des acteurs (figure 1). L'interaction entre les formes et le peuplement par l'intermédiaire de la perception est une notion classique en géographie, qui a été étudiée depuis longtemps à travers les concepts de paysages et de genres de vie. Rechercher des sites pouvant être proposés comme arrondissements culturels c'est rechercher des paysages traduisant une association harmonieuse entre un cadre physique attrayant et une implantation humaine reflétant une adaptation au milieu et une culture originale. Dans cette manière de définir les arrondissements culturels on privilégie avant tous les formes héritées, mais ne constituent-elles pas une composante importante de la culture et de la civilisation d'un pays ? L'objectif de cette recherche

Figure 1

LES TROIS DIMEMSIONS DU PAYSAGE

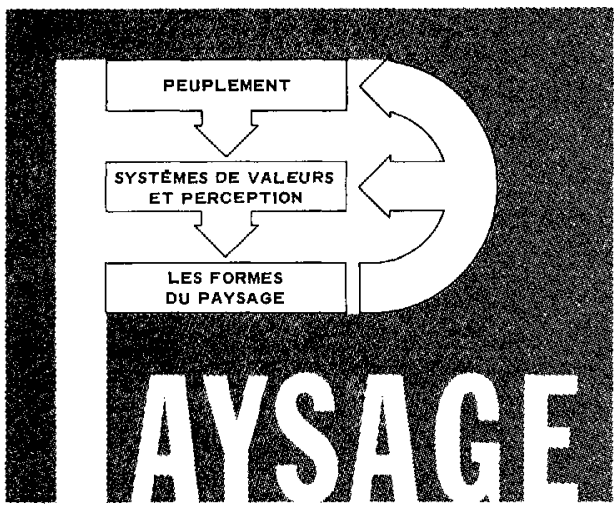

était donc de trouver une méthode permettant de déterminer, à l'intérieur d'une région donnée, les formes héritées dignes d'intérêt et qui sont menacées de disparition à plus ou moins brève échéance par les ruptures d'équilibre engendrées par les progrès techniques du monde contemporain. Une telle approche impliquait une démarche globale, visant à saisir la complexité des caractères culturels propres aux milieux étudiés par le biais d'une analyse à trois volets : les formes, le peuplement et la perception.

Dans le cadre de cet article nous traiterons uniquement de l'analyse morphologique, telle qu'appliquée aux paysages de Charlevoix, les analyses concernant le peuplement et la perception faisant l'objet d'autres articles à l'intérieur de ce numéro ${ }^{4}$. L'analyse morphologique a comme objectif de décrire, et jusqu'à un certain point d'expliquer, les formes visibles des paysages, aussi bien d'après leurs caractéristiques "naturelles » qu'anthropiques. En ce qui concerne les formes d'origine "naturelle», on s'attache à décrire les caractères qui façonnent le cadre visuel des paysages: formes du relief, ampleur des dénivellations, pentes, végétation, réseau hydrographique, caractéristiques écologiques ... Les formes d'origine anthropique traduisent les modifications visibles imposées par l'homme aux paysages primitifs. On les retrouve dans le cadastre, les défrichements agricoles, I'habitat, les voies de communication, l'exploitation forestière, etc. En somme, l'analyse morphologique rend compte des faits visibles (et aussi de certains faits abstraits, invisibles) les plus significatifs qui entrent dans la composition des paysages. 
Mais au-delà de la simple analyse descriptive des paysages, il s'agit d'en établir un classement, une évaluation, permettant de déboucher sur des propositions d'action en vertu de la Loi sur les biens culturels. Examinons maintenant de quelle manière certaines études antérieures peuvent nous être utiles dans la recherche d'une méthodologie appropriée.

\section{L'ANALYSE ET L'ÉVALUATION DES PAYSAGES: UN VIEUX SUJET TRAITÉ PAR DES MÉTHODES RENOUVELÉES}

L'analyse globale des paysages d'une région n'est pas un sujet nouveau en géographie. On peut se référer aux publications de Vidal de la Blache, Jean Brunhes, Max Sorre, Albert Demangeon, ainsi qu'aux grandes thèses régionales françaises publiées depuis 1930, pour constater que la démarche globale prônée par les écologistes modernes était déjà pratiquée depuis longtemps par les géographes français de la première moitié du XXe siècle. Cette appréhension globalisante des paysages était souvent le fruit de longues et patientes recherches s'étalant sur une dizaine d'années ou plus et faisant intervenir une vaste érudition de la part des auteurs. Le but ultime était d'expliquer la genèse et l'articulation des paysages d'une région dans une perspective de connaissance pure. Plus près de nous, depuis une dizaine d'années, des géographes ont été appelés à appliquer leur science à des problèmes concrets touchant l'inventaire des ressources, la désignation de parcs nationaux et d'aires de conservation, des études d'impact de projets d'aménagement sur l'environnement, etc. Pour résoudre ces nouveaux problèmes les géographes ont dû mettre au point des méthodes d'analyse plus expéditives et faisant appel le moins possible à la subjectivité du chercheur ; de plus, on leur a demandé non seulement d'établir des typologies ou des classifications de paysages mais aussi de procéder à des évaluations de la valeur relative de ceux-ci en fonction de critères particuliers de nature esthétique ou purement utilitaire.

La plupart des études récentes relatives à l'analyse des paysages comportent deux volets: une classification des composantes et une quantification de leur "valeur" en fonction de critères particuliers. Dans certains cas les auteurs se limitent à la seule classification alors que d'autres basent leur évaluation sur des études de perception sans avoir effectué de typologie au préalable. Examinons maintenant quelques recherches antérieures qui illustrent ces différentes approches.

\section{Classifications exhaustives privilégiant les caractères bio-physiques}

Les travaux les plus exhaustifs en matière de taxonomie des paysages ont été entrepris par des biogéographes ou des écologistes préoccupés au départ par la cartographie de la végétation. Leur hypothèse de base est que la végétation intègre les variables écologiques prépondérantes (Long, 1974, vol. 1, p. 80) : altitude, pentes, dépôts de surface, drainage, climat, intervention humaine, etc. En cartographiant les unités de végétation à un niveau de perception (échelle) donné on se trouve à résumer en même temps les principales composantes du paysage visible. La carte intégrée de la végétation constitue le document de base à partir duquel il est possible de tirer des évaluations concernant différents types d'utilisation ou d'aménagement d'un 
territoire, dont la préservation de certains paysages d'intérêt remarquable. $C^{\prime}$ est probablement en France que les recherches en matière de cartographie intégrée de la végétation ont été poussées au plus haut point, à Toulouse. sous la direction de $\mathrm{H}$. Gaussen (Service de la carte de la végétation de la France), et à Montpellier dans la lignée des recherches de L. Emberger et J. Braun-Blanquet (Centre d'études phytosociologiques et écologiques). Les publications de $G$. Long (1969 et 1974-75) ainsi qu'un numéro spécial de la Revue géographique des Pyrénées et du Sud-Ouest (1972) intitulé "La Science du paysage " donnent un bon aperçu de ce courant de recherche. Les méthodes de l'école montpellieraine ont été adaptées et appliquées au Canada sur de grandes superficies de territoire par une équipe d'Environnement Canada dirigée par Michel Jurdant. Une cartographie d'envergure a été effectuée dans la région du Saguenay-Lac-Saint-Jean (Jurdant et al., 1972 et 1977 ) et plus récemment sur le territoire de la municipalité de la Baiede-James.

À partir des cartes écologiques intégrées il est possible de tirer des interprétations concernant la nature des paysages d'une région et leur attrait (Jurdant, 1972, p. 62 et 1977, p. 94). Ces interprétations privilégient évidemment les caractères "naturels" et "sauvages" des paysages et n'accordent que très peu de place aux éléments humains. À ce titre elles sont plus appopriées pour la délimitation de parcs nationaux ou de réserves écologiques que pour la reconnaissance d'arrondissements culturels.

\section{Classifications sommaires ou partielles accompagnées d'un jugement de valeur utilitaire}

Ces classifications, effectuées par des spécialistes, ont pour but de classer les paysages ou certains de leurs éléments en fonction d'une utilisation déterminée, qui correspond généralement à diverses formes de récréation de plein air ${ }^{5}$. La classification elle-même est accompagnée d'un jugement de valeur relatif à "l'usage" potentiel des différentes parties du paysage. Un exemple typique de ce genre de classement est constitué par les cartes de potentiel des terres à des fins récréatives levées dans le cadre de l'Inventaire canadien des terres (Canada, 1969). Citons quelques-uns des objectifs de ce programme de classification des terres à des fins récréatives (les italiques sont de nous) :

- " offrir une réelle vue d'ensemble de la qualité, du volume d'utilisation et de l'emplacement des zones propres aux loisirs de plein air dans les régions habitées du Canada:

- déterminer les divers degrés d'aptitude récréative des terres non urbaines en fonction de la demande populaire actuelle;

- déterminer le genre de loisir auquel le terrain se prête le mieux;

- identifier les terrains ou les caractéristiques qui présentent une valeur récréative exceptionnelle ou unique;" (Canada, 1969, p. 3).

D'autres tentatives de classement et de mesure de l'intérêt des paysages ont été appliquées sur des portions de territoire plus réduites. Ainsi, Linton (1968) a tenté de répartir les paysages d'Écosse à l'intérieur de six types 
de "Landform landscapes " et sept types de "Land-use landscapes "; il leur a attribué ensuite une cote différente basée sur l'ampleur des dénivellations, la variété du relief, le caractère "sauvage" des paysages, la diversité de l'utilisation du sol, etc. II a obtenu un classement combiné tenant compte à la fois des éléments naturels des paysages et de l'occupation humaine. II s'agit d'une méthode relativement facile à appliquer, n'exigeant pas un entraînement très poussé et reposant essentiellement sur l'analyse des cartes topographiques et photographies aériennes. Elle a été reprise avec succès semble-t-il, par Gilg (1976) qui considère que la méthode permet d'obtenir des résultats valables sans exiger pour autant beaucoup de travail de terrain. d'analyses de laboratoire ou de calculs statistiques.

La classification des paysages riverains des cours d'eau a fait l'objet de nombreuses études de détail. Ainsi Léopold (1968) a tenté de définir et d'appliquer un " indice de rareté » des paysages à partir de critères chimiques, biologiques et d'occupation humaine. La méthode semble avoir été vivement critiquée en raison du caractère arbitraire des cotes attribuées aux variables et de l'absence de méthode d'échantillonnage dans le choix des sites étudiés. La méthode utilisée par Dearinger (1968), dans le Kentucky, semble plus intéressante du fait qu'elle est beaucoup plus englobante. L'auteur attribue des cotes aux éléments clés du paysage liés à des activités récréatives. Il utilise plusieurs variables que l'on rencontre rarement dans les études du genre: micro-climat, hiérarchie des cours d'eau, éléments de pollution, variables culturelles. Finalement, l'auteur tente de construire une équation prédisant le degré d'attraction des différents sites à partir d'un calcul de régression multiple. Cette étude se distingue des autres par le fait qu'elle combine des mesures esthétiques, la détermination du potentiel d'attraction des sites et l'estimation des bénéfices économiques liés à la demande touristique.

La méthode des calques superposés développée par I. McHarg (1969) mérite d'être citée également en raison de sa simplicité et de son utilisation assez répandue dans plusieurs bureaux d'urbanisme. Appliquée essentiellement pour évaluer l'impact de certains projets d'aménagement du territoire sur l'environnement, la méthode consiste à classer chaque variable considérée en un nombre limité de niveaux, selon le caractère positif ou négatif de la variable en fonction du projet envisagé. Ces niveaux sont traduits par des trames d'intensité (ou de couleur) différente. La superposition des calques correspondant aux variables choisies permet de dégager instantanément les zones plus ou moins favorables à l'implantation de tel ou tel projet. La méthode a l'avantage d'être relativement simple dans son application mais elle implique des jugements de valeur dans le choix et la gradation des variables: le nombre de celles-ci ne peut guère dépasser une dizaine en raison des limitations du système graphique à base de superpositions. La méthode se situe plutôt à l'aval d'un projet, au stade de l'évaluation des paysages, et ne remplace pas les opérations d'inventaires et de classification toujours nécessaires.

Les méthodes précédentes présentent plusieurs caractères communs : elles privilégient les caractères physiques des paysages et n'accordent souvent qu'une place réduite aux facteurs culturels; de plus, lorsqu'elles font appel à des cotes numériques, celles-ci sont attribuées par des expertsi en 
fonction de jugements de valeur propres à leur perception des problèmes de l'environnement ; le point de vue du profane n'est que rarement considéré.

\section{Méthodes d'évaluation de la perception des paysages au moyen de photographies}

On doit à $D$. Lowenthal les premières études introduisant la notion de perception dans l'explication des paysages humanisés (cultural landscapes). Il a montré en particulier comment l'évolution divergente des paysages humanisés de l'Angleterre et des États-Unis était due à des goûts différents en matière de perception esthétique des paysages (Lowenthal, 1964, 1965. 1968). Depuis la fin des années 60, on a vu éclore en Angleterre toute une série de publications relatives aux relations perceptuelles existant entre un observateur et un paysage. Fines (1968) a été l'un des premiers à tenter de mesurer cette relation en montrant à un groupe de personnes un jeu de photographies illustrant divers paysages. Chaque sujet était invité à attribuer une cote aux photographies de paysages par comparaison avec une photographie témoin. La même méthode photographique a été utilisée par Shafer $(1969,1970)$ qui est allé plus loin en mesurant la part relative des différents types de paysages présents sur chaque photographie faisant l'objet de tests de perception.

L'évaluation de l'attrait des différents types de paysages au moyen de photographies soumises au jugement de certains groupes de personnes constitue une méthode commode qui devrait permettre, en théorie, de connaître facilement et rapidement les goûts du public en matière d'esthétique des paysages. La méthode présente cependant de nombreux défauts qui limitent son application: représentativité des photographies, variations dues aux émulsions, caractère artificiel et partiel de l'image photographique par rapport au paysage réel, etc.

\section{Analyses basées sur un modèle symbolique de l'esthétique des paysages}

Jay Appleton, dans son ouvrage intitulé The Experience of Landscape (1975), a tenté de construire un modèle de classification des composantes des paysages basé sur la théorie dite "perspective-refuge " (prospect-refuge). Cette théorie est basé sur l'observation du comportement de l'homme et des animaux qui les pousse à explorer leur environnement de manière à toujours "voir sans être vu». Cette attitude en est une de survivance biologique, vérifiée de manière expérimentale par le zoologiste $K$. Lorenz. Le lieu où un observateur a la possibilité de voir sans contrainte constitue une " perspective " (prospect) ; le lieu où il a la possibilité de se cacher constitue un "refuge". Selon la théorie "perspective-refuge", la possibilité de voir sans être vu est une étape intermédiaire dans la recherche visant à satisfaire de nombreux besoins biologiques ; la capacité qu'a un environnement donné de répondre à cette condition constitue une source de satisfaction esthétique immédiate. Cette théorie fournit à l'auteur un cadre de référence pour une interprétation symbolique des propriétés esthétiques du paysage. Le symbolisme d'un paysage donné est analysé à l'aide de différents termes reliés à la théorie: types de perspectives (directes, indirectes, panoramas, etc.). obstacles, types de refuges (selon leur nature, leur fonction, leur origine), 
texture des surfaces, influence de la lumière, niveaux de symbolisme, échelle, mouvement, etc. Ce type d'analyse n'a pas d'autre but que d'identifier les composantes esthétiques des paysages, sans essai de quantification.

Une quantification de l'esthétique des paysages a été tentée dans le cadre d'un inventaire écologique de l'île d'Anticosti (Québec, 1974). Selon l'auteur de cette recherche, "l'esthétique des paysages réfère au caractère ou à la beauté des aires ou scènes naturelles extensives et aux divers objets qu'elles contiennent ... L'objet d'une étude de l'esthétique de paysage doit se rattacher à une expérience interne et non externe à la zone; quant à la qualité et à la quantité d'appréciation de cette expérience, elle reflètera directement le potentiel récréatif ». Le dynamisme des paysages est également mesuré : il «se rapporte à la vitalité intrinsèque et à l'énergie contenues dans le paysage et demeure indépendant de son appréciation ". Les termes de référence utilisés présentent beaucoup de points communs avec ceux de la théorie symbolique de Appleton. Les fondements de l'évaluation numérique des divers facteurs considérés demeurent cependant arbitraires et difficilement généralisables, d'autant plus que le milieu où la méthode a été appliquée est essentiellement sauvage avec une présence humaine réduite au minimum.

\section{Nécessité d'une approche globale de l'évaluation des paysages culturels}

L'examen des recherches antérieures concernant I'analyse des paysages montre qu'il existe souvent une confusion entre la classification et l'évaluation proprement dite des composantes des paysages. Or, on pourrait dire que les formes des paysages existent indépendamment du symbolisme, des qualités esthétiques ou des fonctions utilitaires qu'on leur attribue. Les systèmes de classification déjà utilisés dans les recherches antérieures accordent une place prépondérante, sinon exclusive, aux formes "naturelles" des paysages ruraux ou forestiers. Pour une région donnée, il importe donc de développer une classification qui assure une meilleure représentation des formes anthropiques, en tenant compte du contexte particulier à la région: échelle, type de relief, ancienneté du peuplement, degré d'urbanisation, etc.

Lorsque vient le temps d'effectuer l'évaluation de la "qualité " ou de "l'intérêt » des paysages, le problème est de savoir qui va faire l'évaluation: sera-ce "un spécialiste " ou un groupe de profanes ? L'idéal serait d'utiliser les deux. Il est bien évident que seuls des spécialistes peuvent apprécier à leur juste valeur une formation géologique rare, un groupement végétal ou animal menacé de disparition, ou une forme agraire typique. Le jugement des spécialistes est indispensable lorsqu'il s'agit de mesurer l'intérêt scientifique de certaines formes de paysages. Par contre, la mesure de l'attrait esthétique des paysages doit faire appel au jugement du profane. Cette mesure pourra être combinée à d'autres formulées par les experts, concernant le degré de rareté des paysages culturels et les menaces de détérioration les affectant. Soulignons cependant qu'une forme typique, ou rare, ou d'un intérêt scientifique remarquable, n'est pas forcément belle et que le jugement esthétique du profane ne saurait en aucun cas être considéré isolément (Matley, 1977. p. 65). 
La première étape indispensable, mais souvent escamotée, de l'évaluation des paysages humanisés d'une région, consiste à dresser un inventaire des morphologies. Avant d'exposer la méthodologie suivie pour effectuer cet inventaire dans la région de Charlevoix, examinons l'intérêt de celle-ci pour une analyse morphologique des paysages.

\section{INTÉRÊT DES PAYSAGES DE CHARLEVOIX POUR UNE ANALYSE DES MORPHOLOGIES}

La région de Charlevoix ${ }^{6}$ est située sur la rive nord du Saint-Laurent, à une centaine de kilomètres au nord-est de la ville de Québec (figure 2).

Figure 2

\section{E R E L I E F D E C H A R L E V O I}

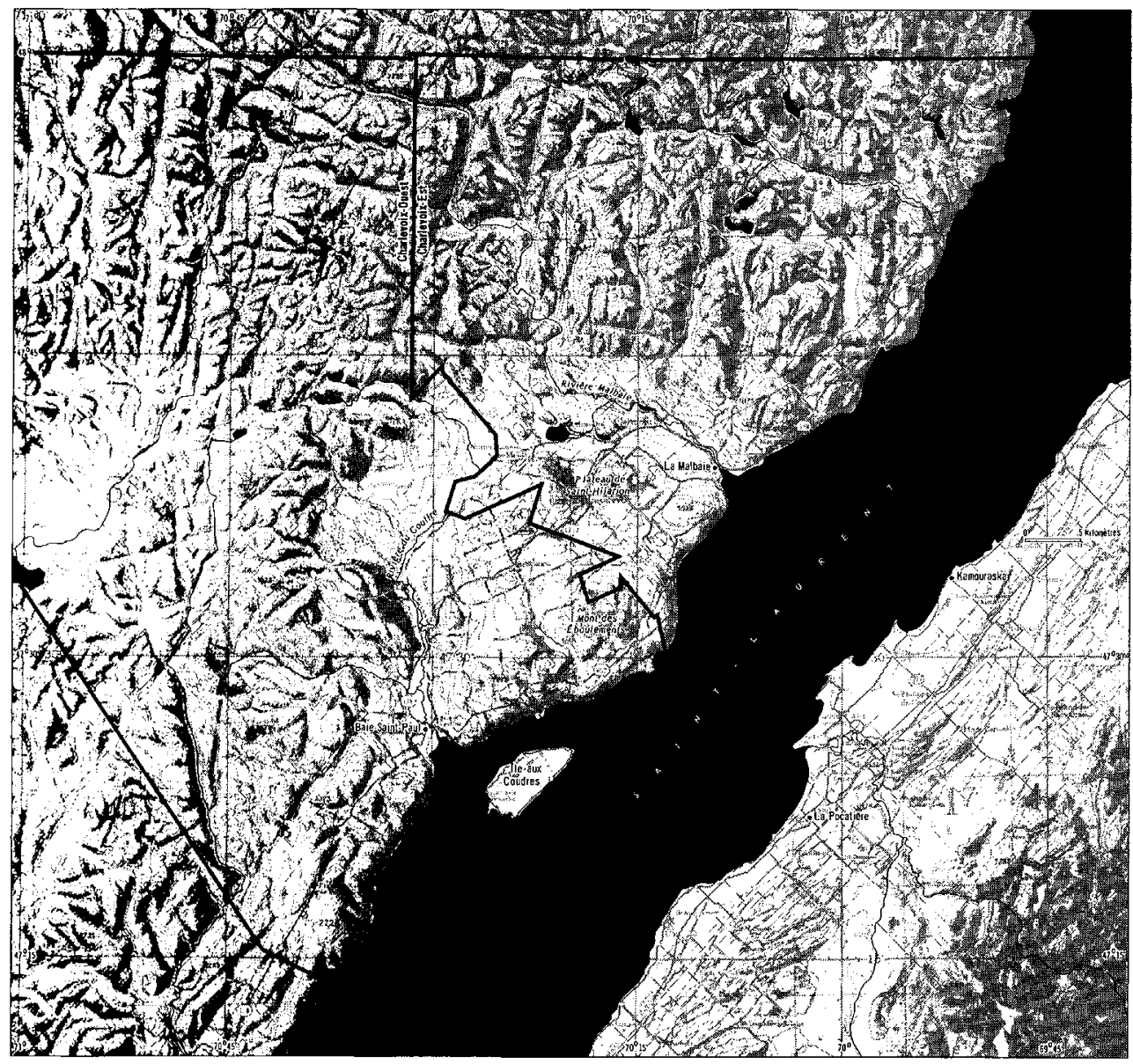


Elle couvre $5700 \mathrm{~km}^{2}$ dont seule une frange côtière variant de 10 à $25 \mathrm{~km}$ est habitée. Il s'agit d'une région montagneuse, fort pittoresque, fréquentée de longue date par les touristes et les peintres ${ }^{7}$.

\section{Des paysages contrastés}

Les paysages de Charlevoix revêtent un caractère exceptionnel du fait qu'ils sont très contrastés et très individualisés sur de courtes distances. Le contraste est d'abord topographique, par la succession, sur une distance de 30 à $40 \mathrm{~km}$, du littoral, des deux vallées des rivières du Gouffre et Malbaie, du plateau de Saint-Hilarion, du piémont périphérique et de sommets culminant à plus de 1000 mètres. Le contraste est aussi géologique, par le contact des roches très résistantes du bouclier précambrien avec les calcaires ordoviciens et les dernières formations des basses terres du SaintLaurent que l'on retrouve à l'île aux Coudres, sur certaines parties du littoral et sur les bords de la vallée du Gouffre. Le phénomène de l'astroblème de Charlevoix (mont des Éboulements et structure annulaire du relief) vient compliquer l'organisation géologique et topographique de Charlevoix en lui conférant un caractère extrêmement original (Rondot, 1970). À la surface de ce bâti rocheux, les formes d'origine glaciaire ou fluvio-glaciaire des plateaux et vallées dominent ou côtoient les dépôts marins de la mer de Champlain qui ont envahi les vallées et tapissé leurs versants.

La végétation, dans ses grandes lignes, suit l'organisation annulaire du relief et son étagement en altitude. La zone de la forêt décidue, confinée à une étroite bande littorale et aux deux vallées, est occupée essentiellernent par l'érablière à bouleau jaune. Dans la zone de la forêt boréale, l'étagernent en altitude sur le plateau et les sommets voit se succéder, du bas vers le haut, la sapinière à bouleau jaune, la sapinière à bouleau blanc, la sapinière à épinette noire et enfin la pessière à Kalmia et Cladonie. Ce qui rend la végétation de Charlevoix intéressante, c'est sa situation de contact à la limite nord de la forêt décidue et à la limite sud de la forêt boréale. Le contact, loin d'être linéaire, épouse les particularités des sites topographiques contrastés, et provoque des juxtapositions de formations très diversifiées dans lesquelles feuillus et conifères alternent sur de courtes distances. L'effet visuel de cette alternance est particulièrement saisissant en automne et au printemps. Le couvert végétal reflète en partie les oppositions climatiques entre la mer et la montagne. Le littoral du Saint-Laurent bénéficie de températures plus clémentes que la surface des plateaux et la saison sans gel y est plus longue. Par contre, l'état du ciel est plus variable sur la côte. et la partie supérieure des vallées (vers Saint-Urbain notamment) semble jouir de conditions d'ensoleillement plus favorables, avec un taux d'humidité moins élevé.

\section{Un condensé de l'histoire du peuplement du Québec}

L'évolution générale du peuplement dans l'ensemble du comté de Charlevoix peut être divisée en cinq périodes de durée inégale (figures 3,4 et 5). De 1672 à 1825 s'effectue le peuplement littoral des seigneuries. Sous le Régime français on assiste à l'occupation littorale des seigneuries des Éboulements, du Gouffre (Baie-Saint-Paul), de l'île-aux-Coudres (SaintLouis) et de Beaupré (Petite-Rivière-Saint-François). Après la conquête anglaise, les seigneurs Fraser et Nairn encouragent le peuplement des 
seigneuries de Mount Murray et de Murray Bay (La Malbaie). Durant cette première phase, seule la partie sud-ouest du comté s'est peuplée de manière substantielle (figure 5). Entre 1825 et 1881 la population double et six nouvelles paroisses sont fondées à partir des paroisses-mères (figure 3 ). Quatre se situent au nord-est (Saint-Irénée, Sainte-Agnès, Saint-Fidèle, SaintSiméon) et la population des premières paroisses déborde vers le nord (Saint-Urbain, Saint-Hilarion).

De 1881 à 1921 on observe une stabilisation relative de la population à un premier sommet. L'accroissement inférieur à 3000 habitants en 40 ans correspond à une stabilisation des paroisses (qui acquierrent le statut civil de municipalité) de l'ouest et à un léger accroissement de celles du nord et de l'est. Les surplus démographiques ont été absorbés par la colonisation du Saguenay-Lac-Saint-Jean (seconde moitié du XIXe siècle), celle de l'Abitibi-Témiscamingue (début du $X X e$ siècle) et l'émigration vers les États-Unis. De 1921 à 1961 la population totale croît à nouveau de 50\% grâce au développement de l'arrière-pays durant la crise économique (NotreDame-des-Monts et Saint-Aimé-des-Lacs), puis aux progrès de l'urbanisation à La Malbaie, Clermont et Baie-Saint-Paul. Dix nouvelles municipalités sont créées par fragmentation des municipalités plus anciennes (comparer les figures 3 et 4 ). Depuis 1961, la population totale s'est stabilisée et affiche une légère décroissance. On peut y voir plusieurs causes: baisse de la natalité, émigration rurale et stagnation économique générale qui affecte les villes et villages, en particulier la partie ouest du comté, la plus ancienne. La partie est dépasse sa voisine depuis 1931 et continue de croitre lentement.

La répartition des formes construites issues du peuplement, vestiges architecturaux et autres, se calque étroitement sur l'histoire de l'occupation du territoire, par la densité et l'ancienneté des bâtiments. C'est le littoral au sud-ouest de Cap-aux-Oies qui est le plus riche en témoins architecturaux du régime français et du XIXe siècle. De Saint-Irénée vers le nord-est, on trouve également une grande quantité de bâtiments du XIXe avec des traces de l'influence anglophone. Les éléments intéressants de l'architecture rurale agricole ne sont pas seulement présents sur la côte, mais aussi dans les vallées et sur le plateau de Saint-Hilarion.

\section{Les activités économiques dans le paysage : ressources primaires et services}

L'occupation humaine du territoire a longtemps reposé sur l'exploitation de trois ressources principales: la mer, l'agriculture et la forêt. De l'activité maritime il ne reste plus grand chose, si ce n'est des quais en ruines, quelques épaves éventrées de goélettes et de riches coutumes qui disparaissent avec les derniers marins. L'agriculture, handicapée au départ par les rigueurs du climat, la rareté des bons sols confinés aux vallées et l'éloignement des marchés, décline rapidement et on ne peut guère espérer la conserver qu'à l'intérieur de certaines aires favorables, au fond des vallées et en quelques endroits du littoral et du plateau, notamment autour de Saint-Hilarion. Les paysages agricoles constituent toutefois une part importante du patrimoine de Charlevoix et il faudrait éviter que la friche, déjà omniprésente, n'envahisse les derniers champs cultivés. Une bonification des meilleures terres agricoles et le reboisement des sols les moins aptes devraient assurer une certaine stabilisation des paysages agraires. 
Figure 3

\section{L'OCCUPATION DU TERRITOIRE DE CHARLEVOIX SEIGNEURIES ET PAROISSES-MÉRES AU MILIEU DU XIXe SIĒCLE}

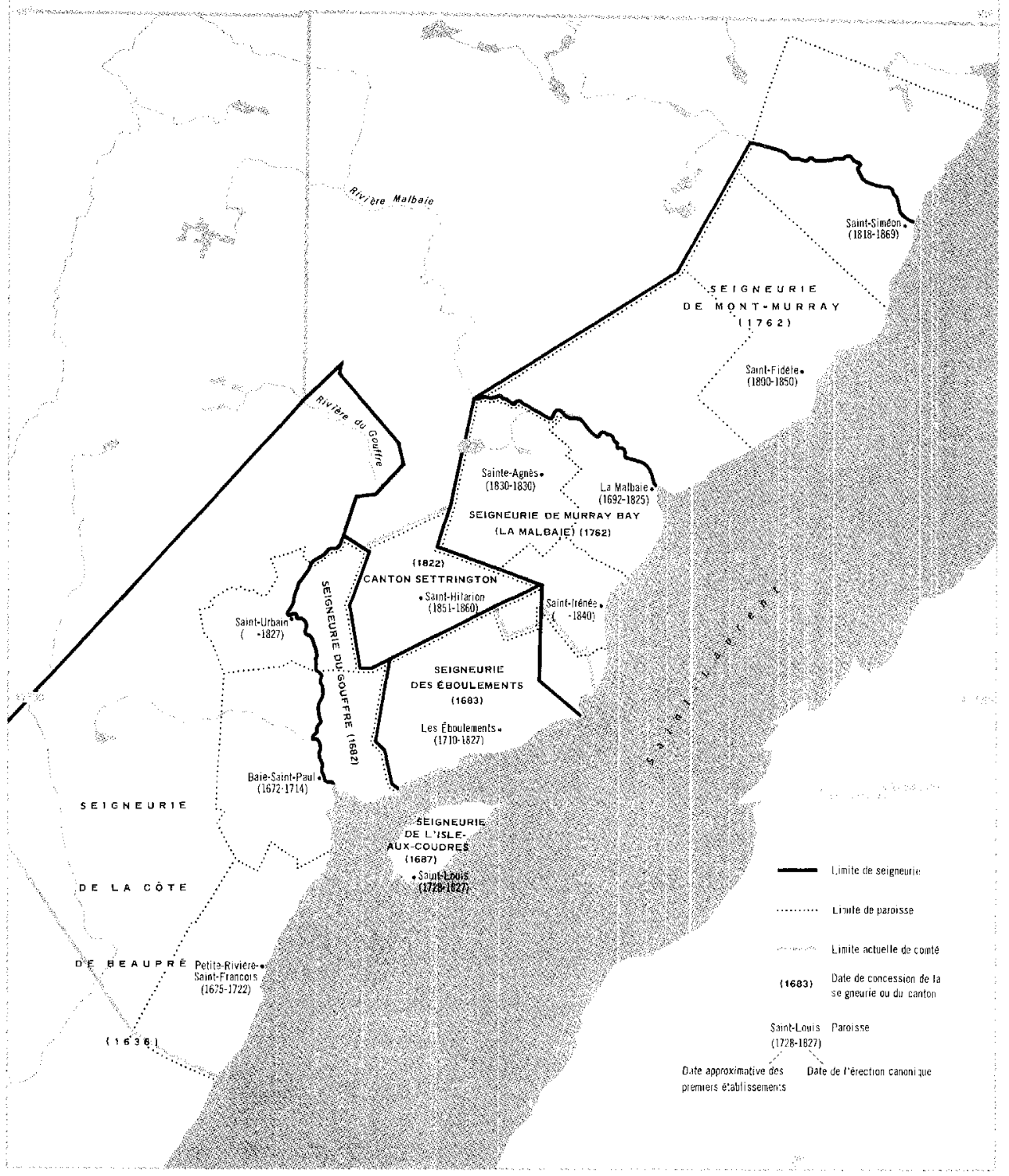


Figure 4

\section{L'OCCUPATION DU TERRITOIRE DE CHARLEVOIX LES MUNICIPALITES EN 1971}

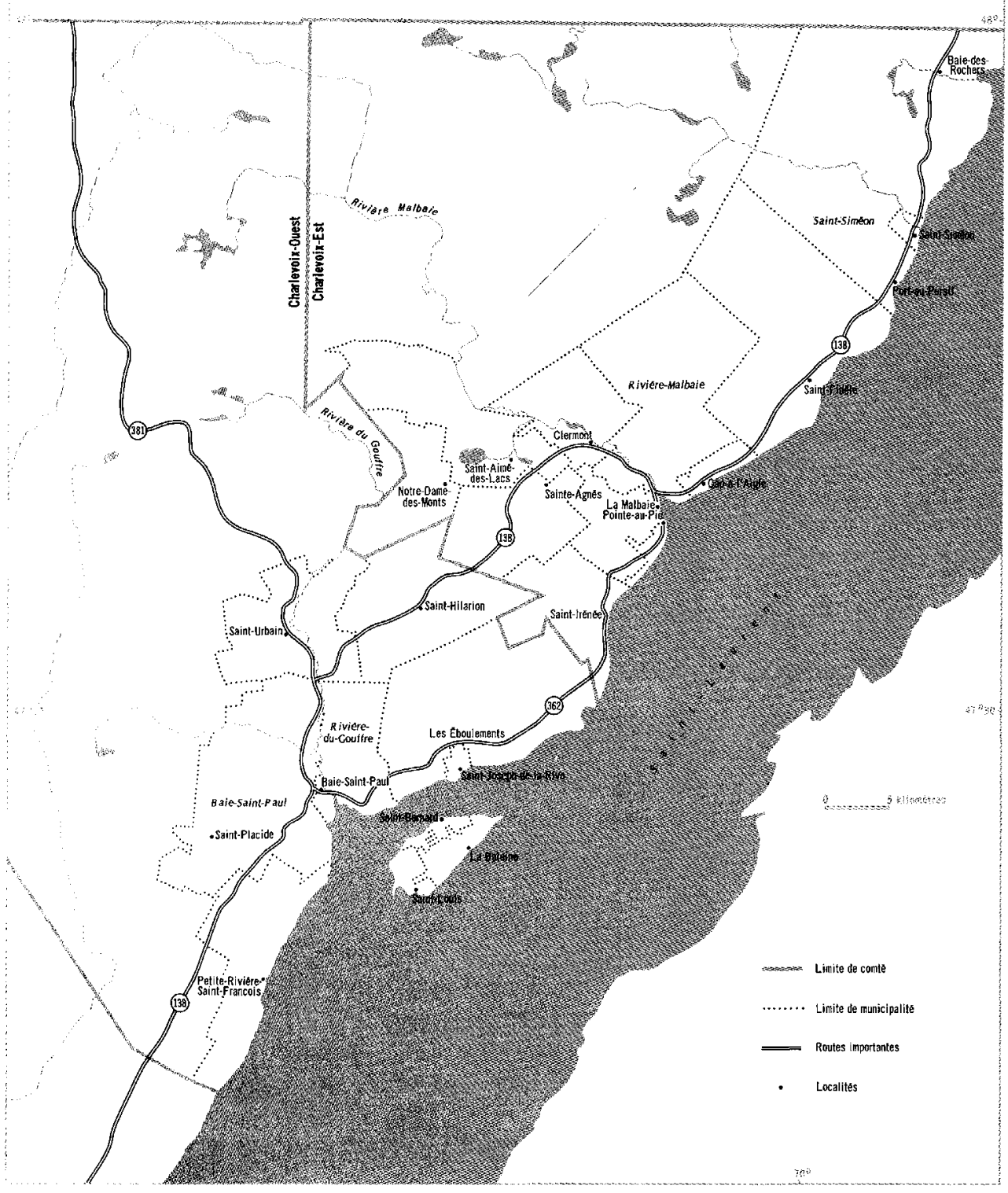


L'exploitation de la forêt a toujours été et demeure encore l'un des piliers de l'économie de Charlevoix. De l'utilisation du pin rouge pour fabriquer le goudron à la fabrication de la pâte à papier (usine Donohue à Clermont) en passant par l'industrie du sciage et la cueillette de la résine, la forêt a toujours constitué et demeure encore une ressource dispensatrice d'emplois et de revenus. L'exploitation des mines s'est soldée jusqu'à maintenant par des espoirs déçus et les perspectives d'un développerment marqué de ce secteur économique sont tout à fait aléatoires et conjecturelles, puisqu'elles sont entièrement soumises à des facteurs extérieurs.

Le tourisme exploite depuis longtemps le caractère exceptionnel des paysages de Charlevoix et la richesse des traditions de ses habitants. Mais jusqu'à maintenant, il s'est agi essentiellement d'un tourisme de passage ou d'un tourisme de séjour de luxe (Manoir Richelieu à Pointe-au-Pic) alimenté par une clientèle riche venant des États-Unis et du Canada anglais. La tendance actuelle est au développement d'un tourisme de séjour populaire que l'on essaie de répartir dans toutes les parties du territoire grâce au développement d'une promotion et d'une structure d'accueil adéquates. L'acquisition récente du Manoir Richelieu par le gouvernement du Québec marque un pas important vers le développement d'un type de tourisme visant un public diversifié et plus proche des habitudes de vie de la population locale.

\section{Figure 5}

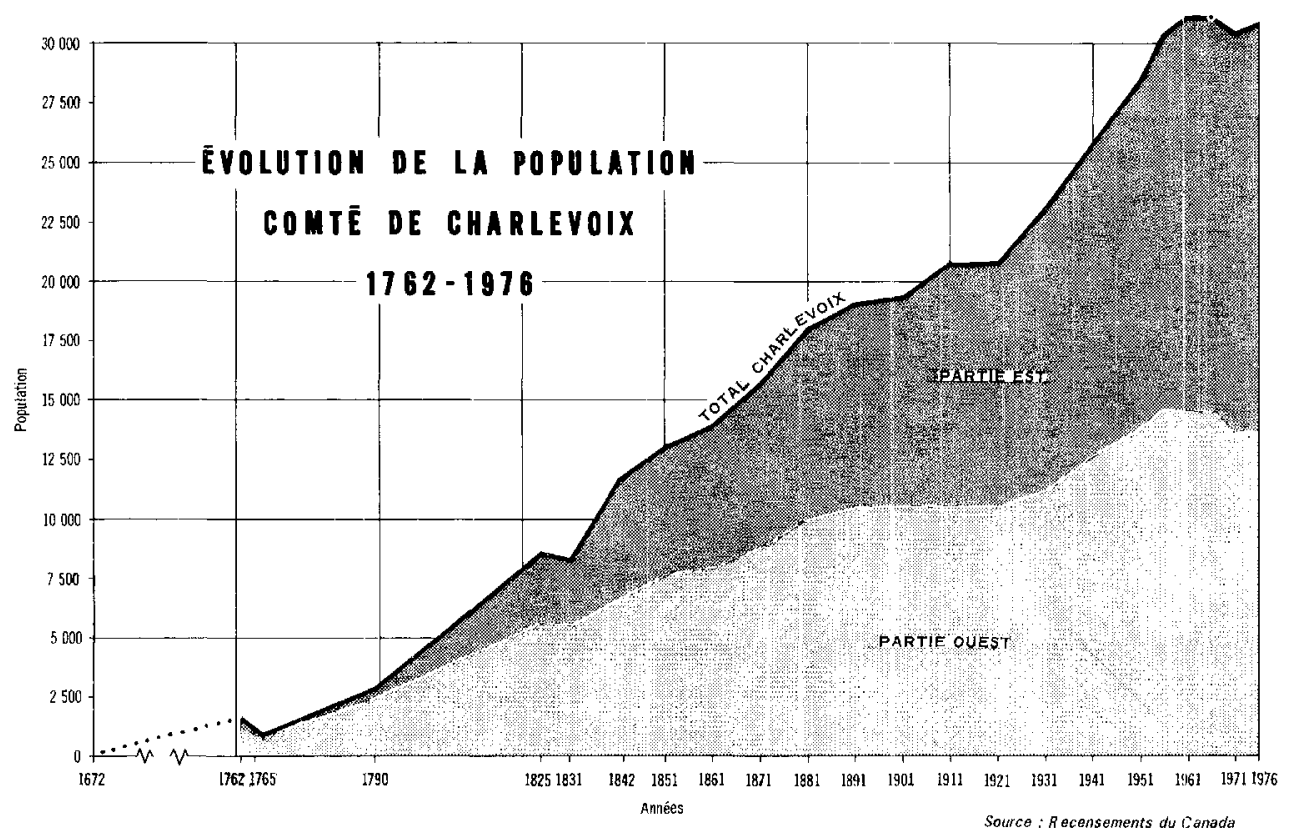


La marque des villes (Baie-Saint-Paul, La Malbaie, Clermont) dans le paysage demeure pour l'instant relativement discrète, d'autant plus qu'elles tirent l'essentiel de leurs ressources des activités de service: commerces, hôtellerie, institutions, administration. Seule Clermont est dominée par une industrie dont l'impact sur l'environnement peut présenter des problèmes sérieux. Mais à moyen et à long terme, l'urbanisation sous toutes ses formes constitue un danger pour la conservation des paysages encore relativement peu pollués de Charlevoix. Une identification des formes actuelles des paysages humanisés est l'étape indispensable à toute action future de conservation.

\section{L'ANALYSE MORPHOLOGIQUE DES PAYSAGES DE CHARLEVOIX: APPROCHE MÉTHODOLOGIQUE}

Avant même d'utiliser une technique particulière pour analyser les paysages de Charlevoix, il est bon de rappeler l'objectif spécifique de la recherche: établir une classification des paysages humanisés en vue de faciliter des décisions ultérieures de protection et de mise en valeur. Cette vision du problème implique au départ que les actions ultérieures de conservation puissent se prendre à diverses échelles, c'est-à-dire que I'on pourra considérer aussi bien de vastes portions de territoires que des zones beaucoup plus réduites, allant jusqu'au simple bâtiment. II importe donc de ne pas se limiter, a priori, à l'identification de zones restreintes ou de sites particuliers, mais de procéder à l'analyse de toutes les parties du territoire. sans préjuger de leur intérêt culturel présumé. À ce stade-ci de la recherche, on doit faire abstraction des formes antérieures d'application de la Loi sur les biens culturels (par exemple à l'île d'Orléans ou à Percé) et considérer la répartition des phénomènes culturels dans l'espace comme un continuum plutôt qu'une série de manifestations isolées.

Avant de juger la valeur de tel ou tel paysage, il importe de le décrire convenablement. C'est pourquoi nous avons procédé en premier lieu à un inventaire des composantes des paysages, puis à une classification. Le travail s'est effectué en deux étapes successives : 1) analyse visuelle des paysages et délimitation des zones d'analyse; 2) constitution d'une matrice d'informations et classification du contenu des divers milieux géographiques de Charlevoix.

\section{L'analyse visuelle des paysages de Charlevoix}

Quand on parcourt une région donnée, on découvre les paysages d'abord par la vision, par une vue globale; la découverte progressive des éléments vient dans un second temps, mais sans perdre de vue l'ensemble et les relations entre les éléments. L'analyse visuelle a comme objectif de décrire la géométrie des formes des paysages et leur agencement dans l'espace. sans nécessairement en établir immédiatement le contenu et la signification. 
Les techniques d'analyse visuelle des paysages ont surtout été développées en milieu urbain ou péri-urbain par des architectes paysagistes. Les méthodes d'analyse des paysages urbains de K. Lynch (1975) sont bien connues et elles ont été reprises dans le cadre d'une étude sur la région de Montréal (Montréal, 1966 et 1971). En milieu rural, Appleton (1975, chap. 4, 5 et 9) fournit des éléments d'interprétation symbolique des composantes visuelles des paysages. Une méthode d'inspiration semblable a été utilisée dans le cadre de l'inventaire écologique de l'île d'Anticosti (Québec, 1974). Aucune de ces méthodes n'était applicable directement à notre objet d'étude et nous avons dû forger nos propres instruments de travail. Dans un premier temps nous avons effectué un relevé systématique et monté un dossier photographique sur toutes les parties accessibles du territoire habité ${ }^{8}$. Ce relevé nous a permis ensuite d'établir une cartographie et une description des éléments suivants: les limites visuelles, les points de vue, les types d'itinéraires.

Le relevé des limites visuelles

La délimitation des périmètres visuels et la localisation des lignes de crête qui les sous-tendent constituent une étape primordiale de l'analyse visuelle des paysages. Elles deviennent en effet un élément important dans la constitution des zones homogènes aux fins de l'analyse spatiale d'une région et elles sont susceptibles ensuite de servir d'élément de référence pour la délimitation de certains sites ou arrondissements culturels. Précisons les termes "ligne de crête " et "périmètre visuel ":

- on appelle ligne de crête la partie la plus élevée d'une forme topographique qui coupe la vue d'un observateur situé en un point donné du terrain :

- on appelle périmètre visuel l'ensemble des lignes de crête qui constituent les limites visuelles d'un paysage; on peut dire aussi qu'un périmètre visuel délimite une zone à l'intérieur de laquelle le paysage observé reste semblable à lui-même (zone homogène).

En fonction de la position occupée par un observateur sur le terrain. l'importance relative des lignes de crête peut varier, selon la proximité et l'altitude des obstacles visuels qui ferment l'horizon. Les formes topographiques les plus élevées et les plus amples constituent des lignes de crête qui coupent la vue de manière continue quelle que soit la position de l'observateur sur le terrain : on les appelle lignes de crête de premier ordre car elles ferment I'horizon et composent l'ossature visuelle qui borde un paysage donné. Constituées par les sommets et les rebords des plateaux les plus élevés, ces lignes de crête de premier ordre forment un fond d'importance régionale sur lequel viennent s'interposer des crêtes de second et de troisième ordre selon leur proximité plus ou moins grande de l'observateur (figure 6 


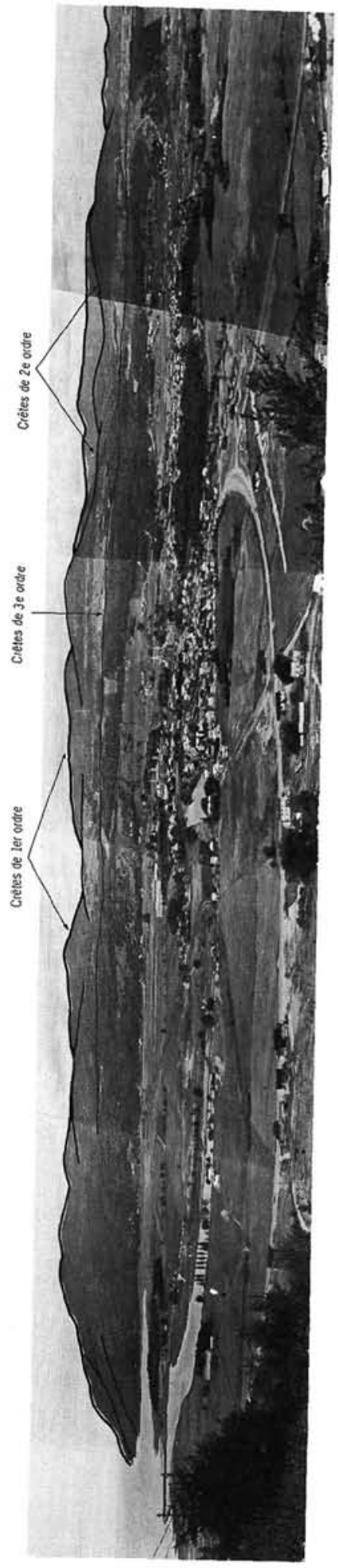

et photo 1). Ainsi, un rebord de terrasse (crête de $3 e$ ordre) constitue I'horizon d'un observateur situé au fond d'une vallée: s'il s'élève quelque peu il découvre une ligne de collines ou un rebord de plateau intermédiaire (crêtes de $2 \mathrm{e}$ ordre), lesquels se détachent sur une ligne de hauts sommets (crêtes de 1 er ordre). Dans Charlevoix, la plupart des lignes de crête du premier ordre se situent sur le rebord du haut plateau des Laurentides à l'ouest et au nord-ouest, ainsi que sur le mont des Éboulements, dans une gamme d'altitudes supérieures à $500 \mathrm{~m}$. Les crêtes de deuxième ordre se situent sur le rebord du plateau de SaintHilarion et des Éboulements, sur le rebord du plateau intermédiaire périphérique et du piémont entre 500 et 150-200 $m$ approximativement. Les crêtes de troisième ordre sont composées par l'ensemble des ruptures de pentes convexes qui se trouvent au-dessous de 150-200 m, généralement sur le bord des vallées du Gouffre et de La Malbaie (figure 6). En cartographiant la position de ces lignes de crête, on parvient à dégager I'ossature régionale des paysages de Charlevoix, premier pas vers l'identification de zones de paysages homogènes (figure $7)$. Les lignes de crête délimitent en quelque sorte des "bassins visuels » à I'intérieur desquels la vue reste semblable à elle-même, quel que soit le point de vue d'observation. 
Figure 6

\section{LE CADRE VISUEL DE CHARLEVOIX LIMITES VISUELLES}

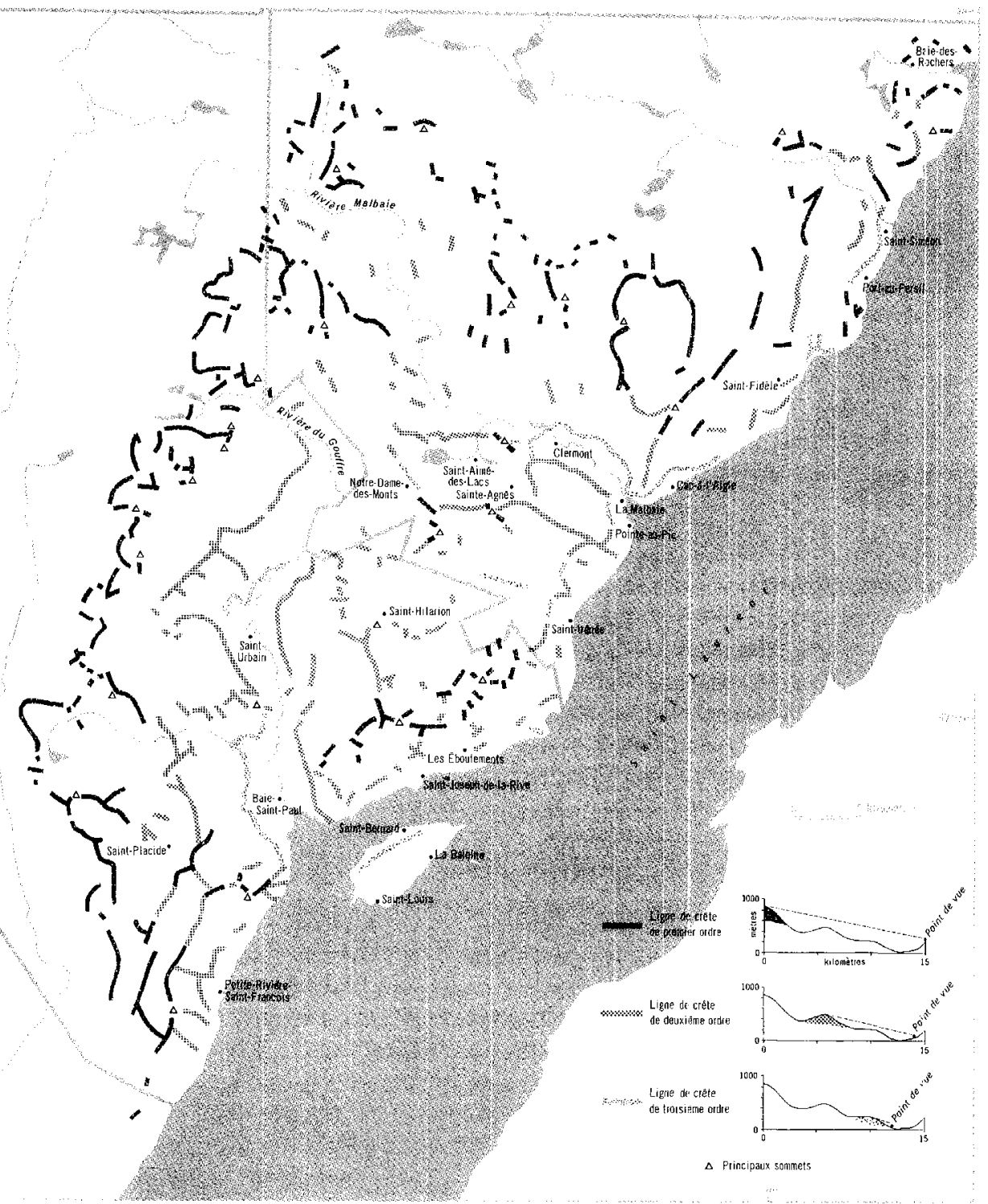


Figure 7

\section{LE CADRE VISUEL DE CHARLEVOIX ZONES DE TRAVAIL}

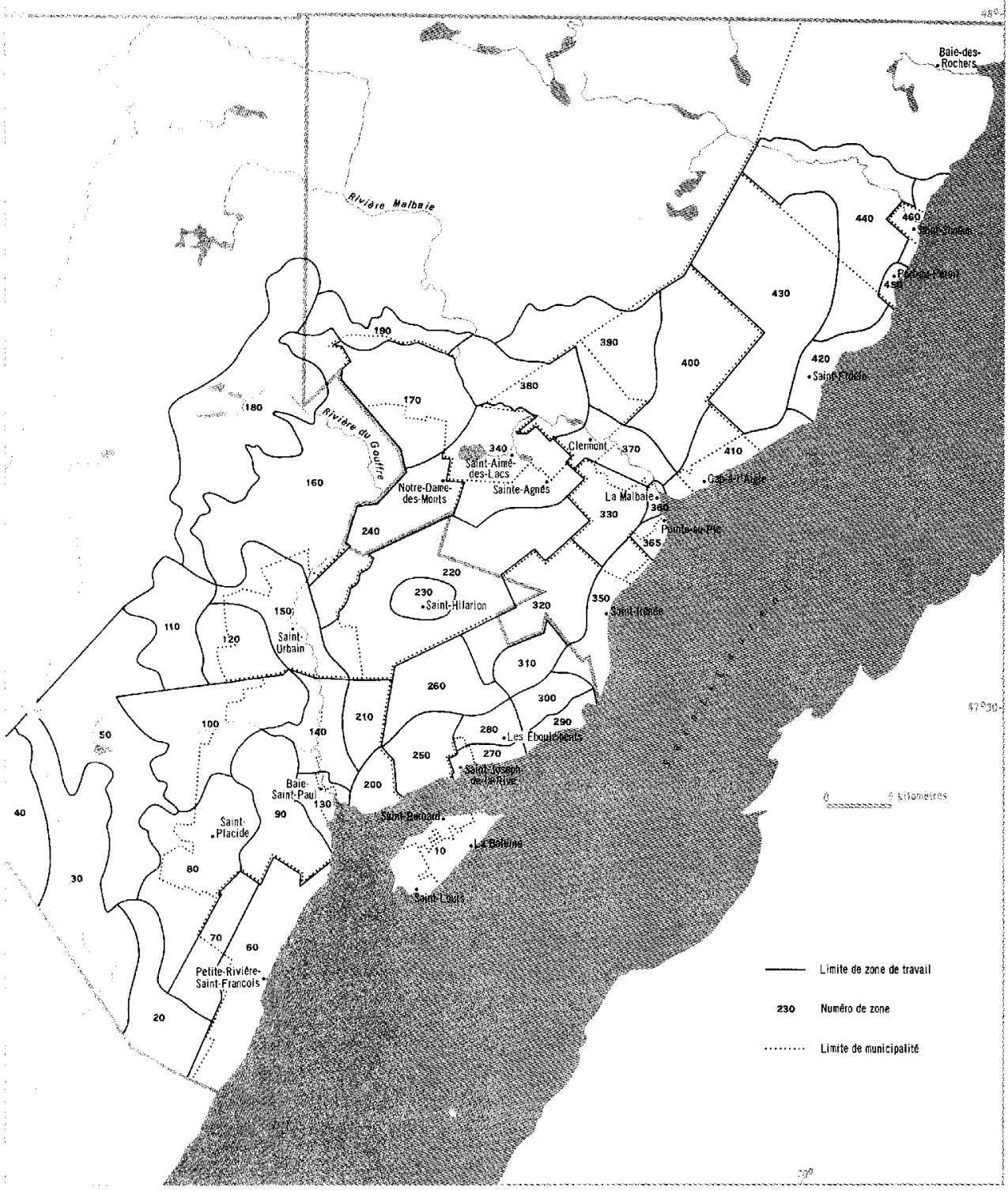


L'identification de ces lignes procède d'une vision de l'intérieur d'un paysage homogène. Si l'on cherche maintenant une vision de l'extérieur, il faudra se placer sur des points de vue ou panoramas permettant d'obtenir une vue circulaire plus ou moins étendue, avec des perspectives horizontales, plongeantes ou ascendantes.

Relevé et classification régionale des points de vue

On a tenté d'établir une hiérarchie régionale des points de vue en tenant compte de la diversité des paysages visibles, de leur distance et de l'angle horizontal du point de vue ${ }^{9}$. Les 101 points d'observation situés sur des routes ou chemins carossables et considérés comme représentatifs des points de vue de la région, ont été mis en relation avec neuf grands types de paysages possédant une combinaison distinctive de traits physiographiques et de formes de peuplement. On a ainsi identifié : la rive sud du SaintLaurent, le Saint-Laurent lui-même, le littoral, l'île aux Coudres, les vallées du Gouffre et de la rivière Malbaie, le plateau de Saint-Hilarion, le plateau intermédiaire périphérique et le piémont, la bordure des hautes Laurentides. Pour tenir compte de la portée de la vue, on a considéré, d'une part, la distance maximale de l'observation en six classes (moins de $10 \mathrm{~km}$, $10-20,20-30,30-40,40-50$ et plus de $50 \mathrm{~km}$ ) et d'autre part l'ouverture approximative de l'angle horizontal du point de vue en trois classes (moins de $180^{\circ}, 180-360^{\circ}$ et $360^{\circ}$ ). Les trois critères ont été combinés en un indice global selon la formule suivante :

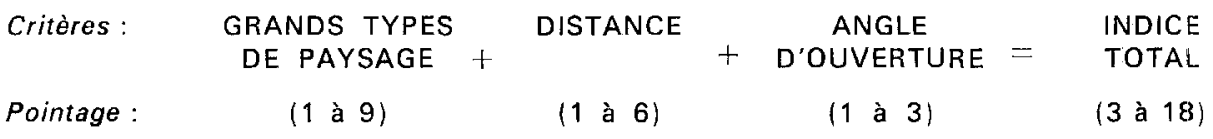

L'analyse des 101 sites de points de vue a permis de faire ressortir quatre types caractérisés par leur indice total et leur localisation : les points de vue régionaux, infra-régionaux, sectoriels et locaux (figure 8 ). Les huit points de vue régionaux (12 à 15 points) permettent de découvrir la majorité des grands éléments physiographiques de la région; à l'exception de ceux de l'île aux Coudres, ils occupent des sites d'altitude qui donnent une perspective plongeante (photo 2). Les points de vue infra-régionaux $(10$ à 12 points) permettent encore de découvrir les grands types de paysages de la région, mais d'une façon plus limitée et moins nette que les points de vue régionaux (photo 3 ) ; ils se subdivisent en points de vue à caractère littoral (généralement des quais) ou intérieur, correspondant aux rebords des deux vallées principales. Les points de vue sectorie/s (6 à 10 points) montrant un nombre limité de grands types de paysages, soit le long du littoral, soit à l'intérieur du plateau, mais rarement les deux à la fois, contrairement aux deux types précédents (photo 4). Enfin, les points de vue locaux (3 à 6 points) ne permettent de découvrir qu'un ou deux grands types de paysages, avec une portée très limitée. C'est généralement l'intérêt du paysage local qui leur donne leur importance (photo 5). Le tableau 1 résume les valeurs moyennes des pointages pour chaque type de point de vue. Ce classement est tout à fait relatif et l'on ne peut pas dire qu'il constitue en soi un jugement de valeur sur la beauté des paysages découverts à partir des points de vue; il s'agit essentiellement d'une distinction d'échelle. 


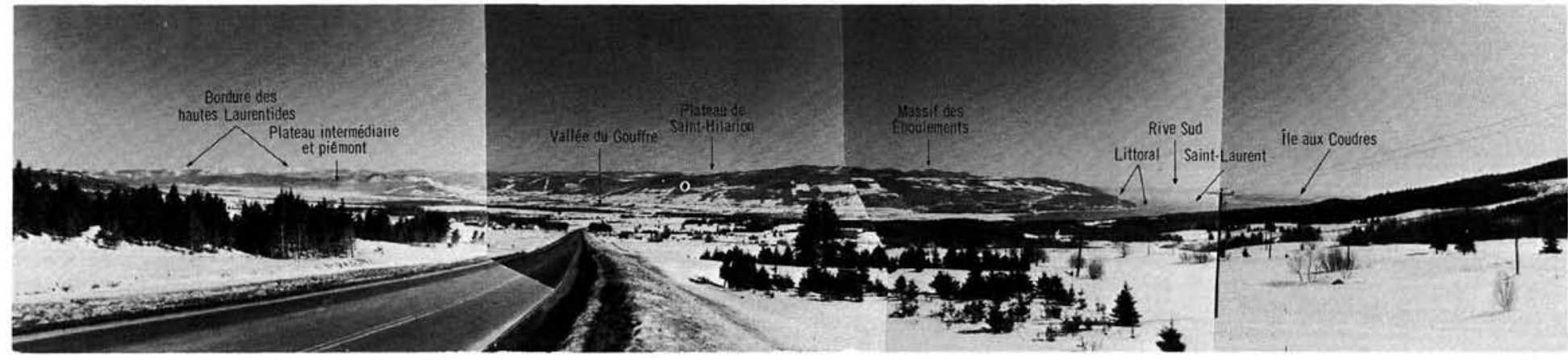

Рното 2 Point de vue régional. Baie-Saint-Paul, rang Saint-Antoine, vue prise vers l'est et le nord, hiver 1975. Distance de vue: plus de $50 \mathrm{~km}$; ouverture: 180 à $360^{\circ} ; 9$ ensembles physiographiques perçus : Saint-Laurent, Rive-Sud, littoral, île aux Coudres, Vallée du Gouffre, plateau de Saint-Hilarion. Massif des Éboulements, plateau intermédiaire et piémont, bordure des hautes Laurentides. Photo Michel Lecarpentier.

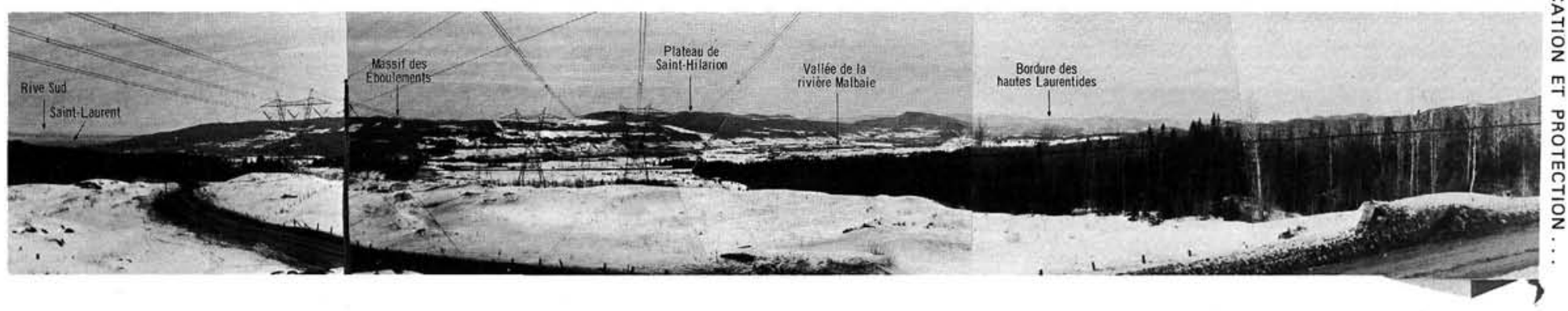

Рното 3 Point de vue infra-régional. Vallée de la rivière Malbaie, vue vers l'ouest, depuis la route de Grand-Fonds, hiver 1975. Distance de vue : $30-40 \mathrm{~km}$; ouverture: 180 à $360^{\circ}: 5$ ensembles physiographiques perçus : Rive Sud, Saint-Laurent, vallée de la rivière Malbaie, plateau de Saint-Hilarion. Massif des Éboulements, bordure des hautes Laurentides. Pollution visuelle des lignes de transmission hydro-électrique. Photo Michel Lecarpentier. 


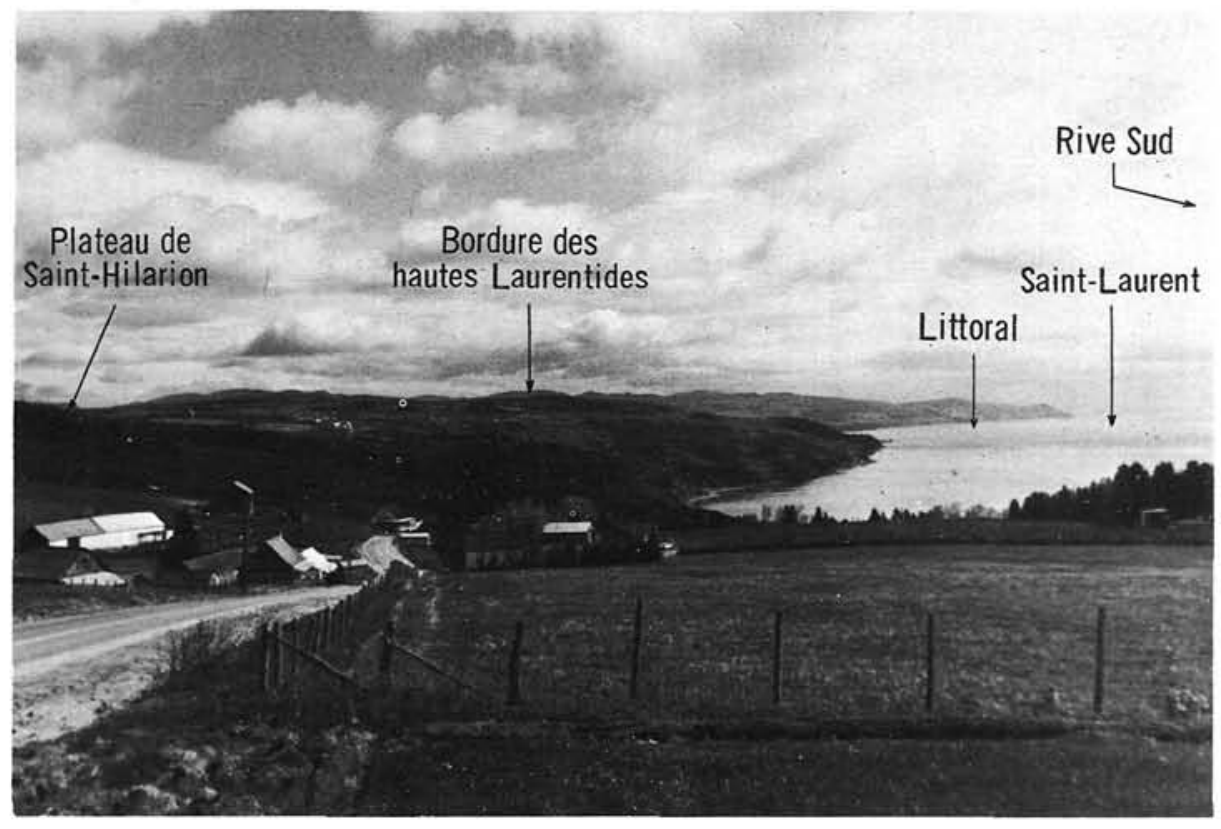

PHOTO 4 Point de vue sectoriel. Saint-Irénée-les-Bains, vue partielle vers le nord-est. Distance de vue: $20-30 \mathrm{~km}$; ouverture: moins de $180^{\circ} ; 5$ ensembles physiographiques perçus: Rive Sud, Saint-Laurent, littoral, plateau de Saint-Hilarion, bordure des hautes Laurentides. Photo Michel Lecarpentier, 1975.

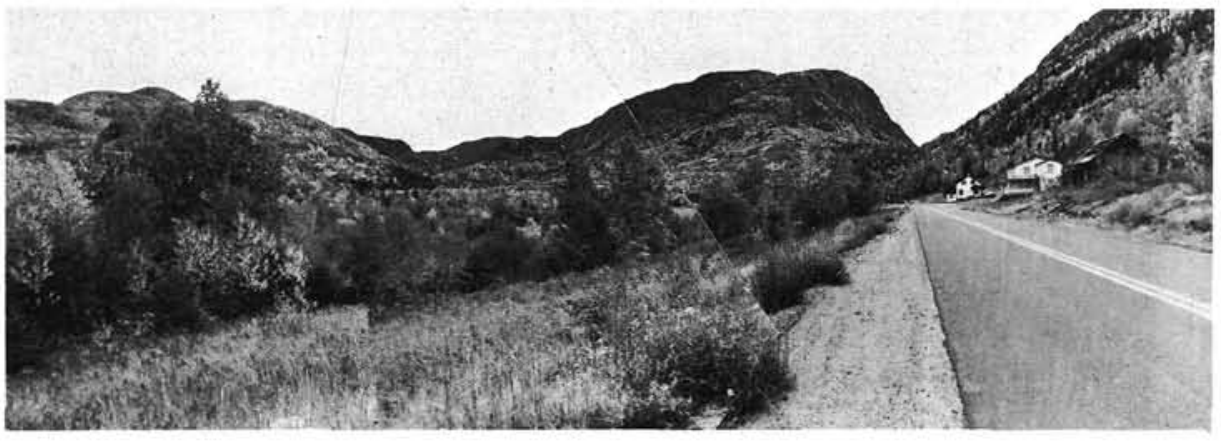

PHOTO 5 Point de vue local. Saint-Urbain, barrière du Parc des Laurentides, route 381. Distance de vue: moins de $10 \mathrm{~km}$; ouverture: moins de $180^{\circ}$; un seul ensemble physiagraphique est perçu: les hautes Laurentides. Photo Michel Lecarpentier, 1975. 
Tableau 1

Pointage moyen obtenu par chaque type de point de vue selon les différents critères d'analyse

\begin{tabular}{|c|c|c|c|c|c|c|}
\hline \multirow[b]{2}{*}{ CRITÈRES D'ANALYSE } & \multicolumn{6}{|c|}{ TYPES DE POINTS DE VUE } \\
\hline & \multirow[t]{2}{*}{ RÉGIONAUX } & \multicolumn{2}{|c|}{ INFRA-RÉGIONAUX } & \multicolumn{2}{|c|}{ SECTORIELS } & \multirow[t]{2}{*}{ LOCAUX } \\
\hline & & Quais & Rebords de vallée & Littoraux & Intérieurs & \\
\hline $\begin{array}{c}\text { Nombre de grands ensembles } \\
\text { de paysages }\end{array}$ & 6,6 & 5,8 & 4,9 & 3,7 & 3,0 & 1,8 \\
\hline Distance maximum d'observation & 3.9 & 3,2 & 4,4 & 3,1 & 2,3 & 1,6 \\
\hline Ouverture du champ de vision & 2,5 & 2,8 & 1,8 & 1,7 & 1,9 & 1,2 \\
\hline Pointage total & 13,0 & 11,8 & 11,1 & 8,5 & 7.2 & 4,6 \\
\hline $\begin{array}{c}\text { Nombre de points de vue dans } \\
\text { chaque catégorie }\end{array}$ & 8 & 6 & 9 & 30 & 23 & 25 \\
\hline
\end{tabular}


Relevé des types d'itinéraires routiers

Le paysage n'est pas appréhendé d'une manière uniquement ponctuelle, à partir de points de vue plus ou moins remarquables. II fait l'objet d'une découverte continue de la part du voyageur qui circule le long d'un itinéraire routier. Chaque section d'itinéraire est en fait constituée de séquences de points de vue qu'il est intéressant de pouvoir classer selon les possibilités de découverte visuelle qu'elles offrent. Parmi les critères de classification des itinéraires, la position de l'observateur par rapport au paysage semble déterminante. Le maximum des possibilités de découverte visuelle des paysages est offert par les sommets de montagnes ou de collines; ceux-ci sont généralement situés en dehors des routes et chemins carrossables. Sur les routes, la position en surplomb, sur le rebord des vallées ou des escarpements, offre le plus de possibilités visuelles si aucun écran ne s'interpose. En position plane, les possibilités d'observation sont conditionnées par l'intensité du défrichement et la présence ou l'absence d'écrans visuels (arbres, maisons). Au fond d'une vallée, le degré d'encaissement, la forme et la largeur de la vallée conditionnement l'étendue de la vue.

Tableau 2

Classification des types d'itinéraires routiers

\begin{tabular}{|c|c|c|c|}
\hline \multirow[b]{2}{*}{$\begin{array}{c}\text { Dégagement } \\
\text { visuel }\end{array}$} & \multicolumn{3}{|c|}{$\begin{array}{l}\text { POSITION DE L'OBSERVATEUR } \\
\text { PAR RAPPORT AU PAYSAGE }\end{array}$} \\
\hline & En surplomb & $\begin{array}{c}\text { En position } \\
\text { horizontale, } \\
\text { relief peu accidenté }\end{array}$ & $\begin{array}{c}\text { En position concave } \\
\text { (en dépression) } \\
\text { relief accidenté }\end{array}$ \\
\hline $360^{\circ}$ & $\begin{array}{l}\text { 1. position } \\
\text { de sommet }\end{array}$ & $\begin{array}{l}\text { 4. plaine, plateau } \\
\text { ou large fond de } \\
\text { vallée complè. } \\
\text { tement déboisé }\end{array}$ & \\
\hline $180-360^{\circ}$ & $\begin{array}{l}\text { 2. rebord de } \\
\text { versant, vue } \\
\text { très dégagée }\end{array}$ & $\begin{array}{l}\text { 5. idem, avec } \\
\text { quelques obstacles } \\
\text { visuels }\end{array}$ & \\
\hline Moins de $180^{\circ}$ & $\begin{array}{l}\text { 3. rebord de } \\
\text { versant, vue } \\
\text { peu dégagée }\end{array}$ & $\begin{array}{l}\text { 6. idem, avec } \\
\text { beaucoup d'obsta- } \\
\text { cles visuels }\end{array}$ & \\
\hline $\begin{array}{l}\text { Dénivellation } \\
\text { relative des } \\
\text { versants } \\
\text { plus de } 200 \mathrm{~m}\end{array}$ & & & $\begin{array}{l}\text { 7. Vallée très } \\
\text { encaissée et } \\
\text { étroite }\end{array}$ \\
\hline moins de $200 \mathrm{~m}$ & & & $\begin{array}{l}\text { 8. Vallée peu } \\
\text { encaissée ou } \\
\text { paysage vallonné }\end{array}$ \\
\hline
\end{tabular}

10. Vue fermée par la végétation, la topographie ou l'habitat, quelle que soit la position de l'observateur

Note: les chiffres sont seulement des numéros d'ordre auxquels on réfère dans le texte : ils n'ont aucune valeur de pondération. 
L'ampleur de la vue est un second critère qui peut se mesurer par l'ouverture du champ de vision et la distance maximum des parties les plus éloignées du paysage visible (déjà mesurée dans l'analyse des points de vue).

Les composantes du paysage local se retrouvent à travers la topographie, la végétation, l'utilisation du sol et l'habitat, tels qu'on peut les observer sur les cartes topographiques et/ou d'utilisation du sol.

En combinant la position de l'observateur et l'ouverture du champ de vision, on obtient plusieurs possibilités théoriques de classification des types d'itinéraires (tableau 2). Sur une carte, on peut ensuite associer les composantes du paysage local à la représentation des types d'itinéraires.

Les itinéraires de Charlevoix (figure 9)

Les itinéraires en surplomb (classes 1,2,3) offrent les meilleures possibilités de découverte visuelle des paysages. La classe 1 est pratiquement absente du fait qu'il n'y a pas de route qui escalade des sommets. Les itinéraires de type 2 , coincidant avec les paysages les plus spectaculaires, se retrouvent sur les versants à l'ouest de la vallée du Gouffre, au nord et à l'ouest du plateau de Saint-Hilarion, sur les hauteurs du littoral aux alentours du cap au Corbeau, des Éboulements, de Cap-à-l'Aigle, et sur la route ceinturant l'île aux Coudres. Les itinéraires de type 3 sont caractéristiques des paysages de plateau et du littoral en aval des Éboulements : ils marquent souvent des zones de transition, des seuils, entre des types de paysages différents.

Les itinéraires horizontaux sont relativement peu nombreux dans Charlevoix. Les plus dégagés (type 4) se retrouvent au nord de Saint-Hilarion et dans la partie nord-est du plateau intermédiaire, alors que les moins dégagés (type 5) caractérisent certaines sections du littoral.

Les deux vallées principales sont évidemment le lieu privilégié des itinéraires encaissés en dépression (types 7 et 8 ). Mais on les observe aussi lorsque la route longe l'estran, au pied du littoral, à Petite-Rivière-SaintFrançois ou à Saint-Irénée. Sur le plateau, les routes empruntant des paysages de hautes collines déterminent également des paysages encaissés.

Les itinéraires fermés (type 10) sont observables surtout à la surface du plateau quand la forêt borde directement la route dans un relief plat ou mollement ondulé. Ils sont typiques également des zones d'habitat dense, des villes et des villages.

En comparant les types d'itinéraires avec l'utilisation du sol, on constate que les itinéraires en surplomb (types 2 et 3 ), de même que les itinéraires horizontaux très dégagés (type 4) sont associés essentiellement aux zones en cultures et secondairement en friches. Les itinéraires encaissés (7 et 8) se partagent assez également entre les zones de friches et de cultures. Les itinéraires fermés (10) dominent évidemment en milieu forestier et dans les zones d'habitat aggloméré. À partir de ces observations on peut affirmer qu'un abandon progressif des zones cultivées dans Charlevoix conduirait inévitablement à une diminution des possibilités de découverte visuelle des plus beaux panoramas de la région, par la «fermeture visuelle » d'un bon 
Figure 8

\section{LA DÉcOUVERTE VISUELLE DU PAYSAGE DE CHARLEYOIX POINTS DE VUE}

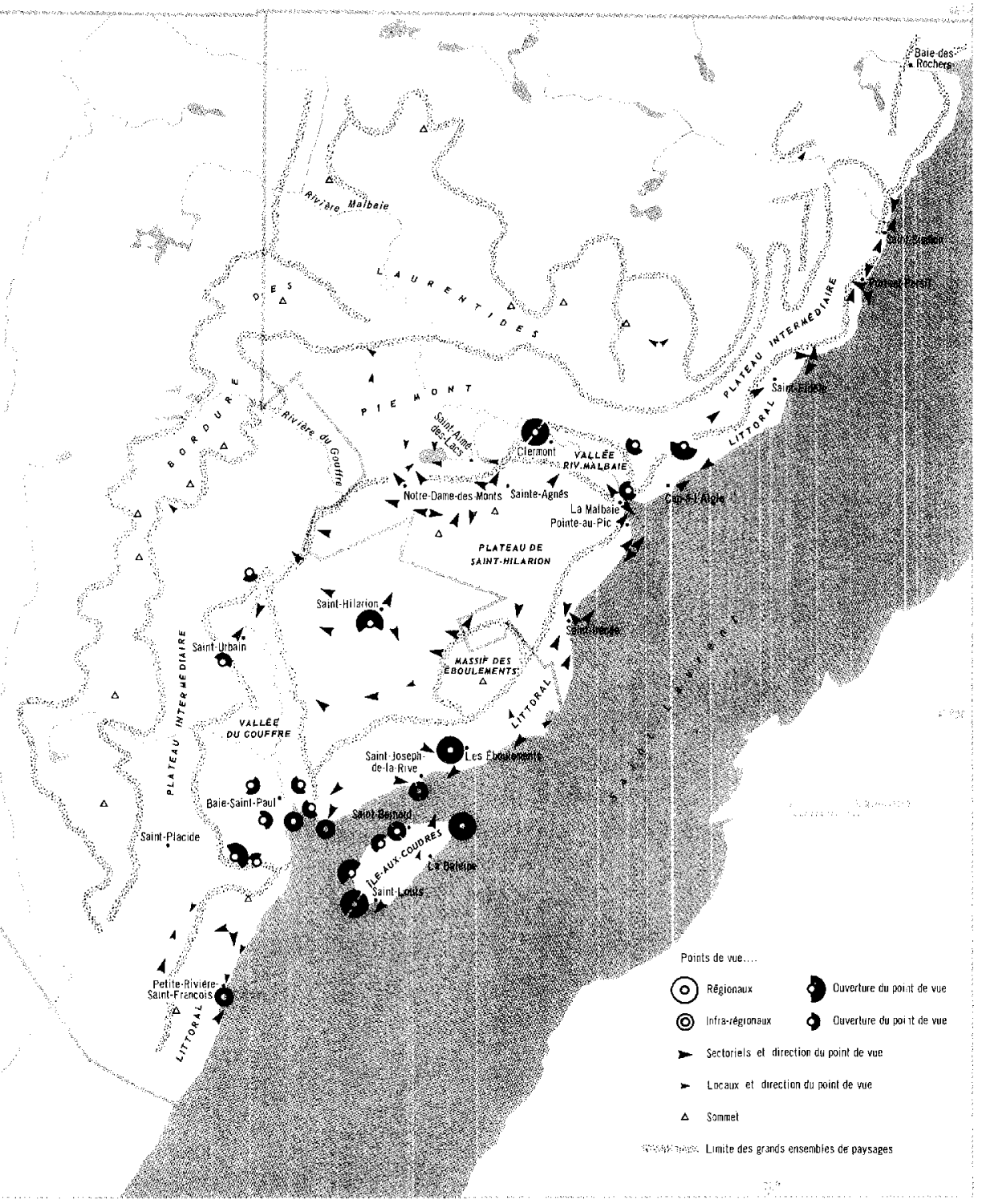


Figure 9

\section{LA DECOUVERTE VISUELLE DU PAYSAGE DE CHARLEVOIX TYPES D'ITINERAIRES}

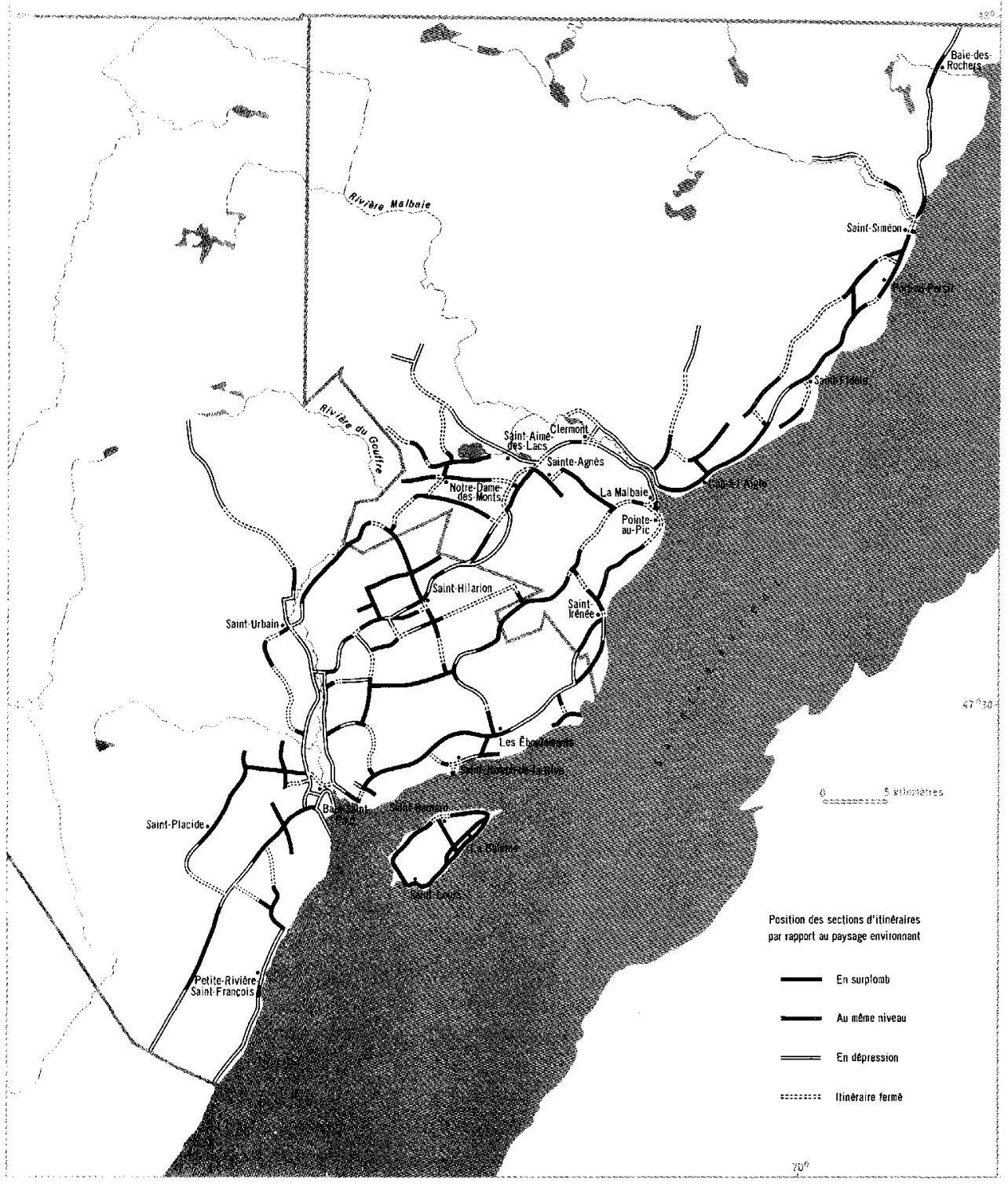


nombre de perspectives que l'on peut découvrir le long des itinéraires routiers.

L'analyse visuelle des paysages permet de systématiser une approche basée sur les sens de l'observateur. Elle permet d'isoler certains paramètres déterminants de la perception. Mais elle est nécessairement limitée quant au nombre de facteurs considérés. Une approche plus exhaustive exige un inventaire détaillé des diverses composantes des paysages, qu'elles soient visibles ou non.

\section{Inventaire spatial et constitution d'une matrice géographique d'informations}

La simple comparaison ou superposition d'un grand nombre de cartes de répartition de variables est insuffisante pour mettre en valeur les relations qui existent entre ces variables et leurs combinaisons spatiales. C'est pourquoi il nous a paru indispensable de monter une matrice d'informations à deux dimensions, constituée de $n$ variables et de $x$ unités géographiques, qui permette de connaître instantanément le contenu de chacune des parties du territoire et qui autorise un traitement exhaustif de l'information à l'aide de méthodes multivariées (matrice visuelle de Berlin ou analyse factorielle).

\section{Montage de la matrice d'informations}

Un premier problème réside dans le choix des unités géographiques devant servir de cadre spatial au relevé des variables. Dans Charlevoix, nous avons essayé de faire coïncider ces unités avec des zones de paysages homogènes bornées par des limites visuelles et présentant des caractères homogènes en termes de topographie, végétation, utilisation du sol, occupation humaine, etc. La dimension des 47 unités d'analyse couvrant le territoire municipalisé de Charlevoix varie de 3,5 à $150 \mathrm{~km}^{2}$ (médiane $30 \mathrm{~km}^{2}$, moyenne $50 \mathrm{~km}^{2}$ ) selon l'intensité de l'occupation humaine ou le degré de fragmentation des paysages. Toutes ces zones homogènes ont une dimension inférieure ou égale à celle des municipalités mais leurs limites ne recoupent pas les limites municipales: il est ainsi possible, pour certaines analyses, d'effectuer des agrégations de zones au niveau municipal.

Le second problème consiste à choisir les variables à inclure dans la matrice d'informations en prévision d'analyses visant à déterminer "l'intérêt esthétique, légendaire ou pittoresque que présente I'harmonie naturelle d'un territoire particulier" (Loi 2). On ne préjuge pas au départ de l'utilisation ultérieure de certaines parties du territoire pour une activité particulière comme c'est le cas lors de relevés concernant le potentiel récréatif par exemple. Ce qui importe en premier lieu c'est de dégager les variables qui structurent l'image des paysages et qui en conditionnent l'évolution, à la fois dans l'espace et dans le temps. En conséquence, nous avons choisi les variables de l'inventaire des morphologies en fonction de leur rôle dans la structuration des paysages, leur pertinence pour les analyses du peuplement et de la perception, leur facilité d'accès au niveau de la compilation. En outre, l'acquisition des variables s'est effectuée de deux manières: d'une part les compilations sur des cartes, tableaux statistiques, rapports divers, et d'autre part les relevés sur le terrain, notamment pour les variables visuelles globales. Les 70 variables ainsi rassemblées (tableau 3) peuvent être divisées en trois groupes: 
- les variables du milieu physique (24)

- les variables de peuplement contemporain (17)

- des variables aléatoires (29) comprenant des variables physiques liées à la côte ou très dispersées (chutes, gravières, etc.) et des variables historiques et socio-culturelles.

Sur les 24 variables physiques, 19 sont présentes dans toutes les zones d'observation. Onze zones ne possèdent pas de population, donc pas de variables de peuplement. Enfin, les variables aléatoires ne sont généralement présentes que dans le tiers à la moitié des zones. La comparaison de toutes les variables à l'aide des valeurs brutes originales s'est avérée pratiquement impossible en raison de l'hétérogénéité des systèmes de mesure: mesures nominales (présence ou absence), par repérage (dates), valeurs absolues, pourcentages, etc. Pour effectuer des manipulations et des comparaisons, il a donc fallu "standardiser » les valeurs, c'est-à-dire les ramener à une échelle commune qui permette la comparaison de toutes les variables, indépendamment du niveau de mesure initial. Toutes les mesures autres que nominales ont été ordonnées en rangs en six classes, d'après l'histogramme de fréquence des valeurs. Nous avons en effet estimé que le niveau de précision de nos mesures rendait illusoire un découpage plus raffiné. Chaque fois que possible nous avons pris la médiane comme seuil central (entre les classes 3 et 4 ) et tenu compte des discontinuités dans la répartition des valeurs pour fixer les autres seuils. De cette manière, il existe une certaine conformité entre l'étendue des classes et les groupements significatifs de valeurs d'une variable.

Méthodes de traitement de la matrice d'informations en vue de l'analyse synthétique des morphologies.

Deux méthodes de traitement étaient à notre disposition pour interpréter le contenu de la matrice d'informations : la matrice visuelle de Bertin (dominos) et les programmes d'analyse multivariée (analyse en composantes principales) à l'aide de l'ordinateur. Ces derniers étaient d'emblée difficiles à appliquer étant donné le grand nombre de valeurs nulles (variables absentes) dans beaucoup d'unités spatiales. C'est pourquoi nous avons préféré au départ manipuler la matrice d'informations selon la méthode de Bertin. L'analyse en composantes principales a cependant été effectuée également à titre de comparaison.

La matrice ordonnable de Bertin

Le principe de base de la matrice visuelle du professeur Bertin (Bertin, 1974) est d'établir une correspondance visuelle entre l'ordre des valeurs à l'intérieur d'une variable et sa représentation dans la matrice. Cette matrice est constituée de dominos perforés, chacun correspondant à une cellule de la matrice (variable yj, unité spatiale $\mathrm{xj}$ ). Les deux dimensions du plan sont donc occupées par $n$ colonnes $(x)$ d'unités géographiques et $n$ lignes $(y)$ de variables. La troisième dimension ( $z i j$ ) correspond au niveau de mesure de la variable yj dans l'unité spatiale $x j$. Elle est traduite graphiquement par une variation de taille et de valeur (au sens graphique) allant du blanc au noir en passant par différentes gammes de gris. Il est ainsi possible de transcrire des séries statistiques en onze paliers différents. Pour notre recherche, 
Tableau 3

\section{Liste des variables utilisées dans la matrice d'informations (sur la base des 47 zones de travail)}

Numéro de

la variable

Variables

\section{A. MILIEU PHYSIQUE \\ Topographie}

1 Altitude maximum

2 Altitude minimum

3 Dénivellation totale

\section{Climat}

4 Température estivale

5 Ensoleillement estival

6 Précipitations estivales

\section{Hydrographie et hydrologie}

7 Nombre de lacs

8 Superficie des lacs

$9 \%$ superficie en eau/superficie totale

10 Longueur des cours d'eau

11 Densité du drainage

12 Superficie des zones marécageuses

16 Chutes ou cascades

Le littoral du Saint-Laurent

13 Longueur de côte

14 Hauteur de l'escarpement

15 Largeur des battures

\section{Géomorphologie}

100 Total des formes observées

101 Total des types de formes

\section{Végétation}

$19 \%$ de la superficie en boisé

$20 \%$ de résineux

$21 \%$ de mélangés

$22 \%$ de feuillus

23 Indice de diversité

18 Superficie totale de la zone

\section{B. PEUPLEMENT ET OCCUPATION DU SOL}

Evolution du peuplement

54 Date de première occupation

52 Repartition de la population 1831

53 Evolution de la population 1831-1971

24 Répartition de la population 1971

25 Densité de la population 1971

53a Evolution de la population 1966-1971

Structure des âges, 1971

55 a Moins de 20 ans, \%

55 b De 20 à 65 ans, $\%$

55 c Plus de 65 ans, \%

Le cadastre

28a Seigneuries ou cantons

26 Profondeur moyenne des lots

27 Largeur moyenne des lots

28 Indice d'allongement des lots
Numéro de

la variable

\author{
Variables
}

PEUPLEMENT ET OCCUPATION DU SOL (suite)

\section{L'utilisation du sol}

$43 \%$ de la surface densément bâtie

$44 \%$ de la surface défrichée

$44 \mathrm{~b} \%$ de la surface en friches

45 a Potentiel agricole classes 2 et 3

45b Potentiel agricole classe 4

45c Potentiel agricole classe 5

Voies de communication

29 Longueur de routes pavées

30 Longueur de routes non-pavées

31 Longueur totale des routes

32 Densité des routes

33 Chemin de fer (présence)

34 Quais (présence)

35 Traversier (présence)

Inventaire architectural

36 Nombre de bâtiments anciens

37 Bâtiments d'intérêt historique et autre

38 Monuments classés

39 Musées

40 Lieux historiques

41 Moulins

42 Centres d'Art

Industries

46 Nombres d'industries

46a Nombre d'employés dans l'industrie

Utilisation des ressources naturelles

47 Mines, carrières, gravières

48 Coupes forestières

49 Brûlis

Loisirs

50 Equipements et attraits divers

51 Equipements sportifs

\section{ANALYSE VISUELLE}

60 Points de vue

90 Types d'itinéraires

\section{PERCEPTION}

70 Lieux représentés par les peintres

80 Perception totale

81 Perception des paysages

82 Perception de l'expression populaire 


\section{REPRÉSENTATION VISUELLE DES VALEURS DANS LA MATRICE DE BERTIN}

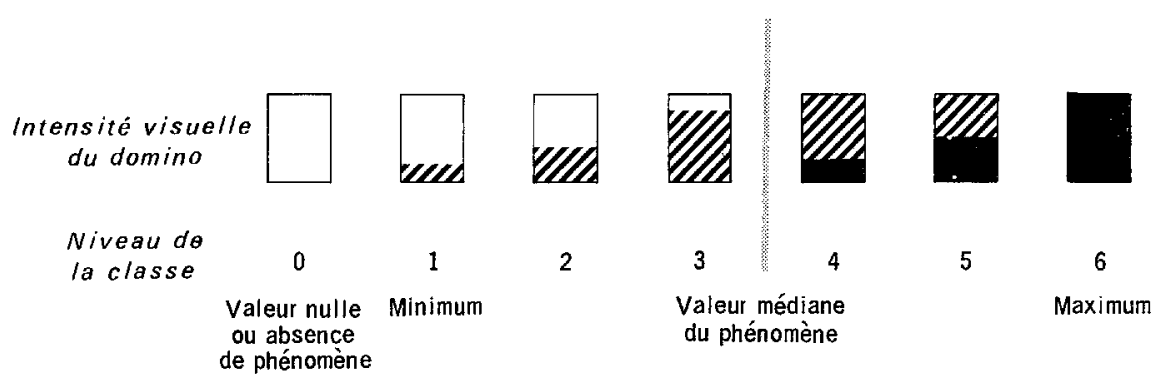

seulement six paliers ont été retenus, auxquels on a fait correspondre six dominos teintés proportionnellement au niveau de chaque classe (figure 10). A partir du tableau donnant le niveau de chaque variable pour chaque unité spatiale, on a "monté " la matrice en remplaçant chaque case du tableau par le domino approprié. Les dominos sont troués sur quatre faces de manière à permettre une enfilade, à l'aide d'une tringle, selon les lignes ou les colonnes. Il est ainsi possible de permuter les lignes et les colonnes, une par une ou par blocs. Les permutations permettent ainsi de rapprocher les lignes ou les colonnes qui sont visuellement (donc statistiquement) semblables. On peut ainsi procéder à des groupements de variables qui ont une répartition statistique semblable à travers certaines unités géographiques, ou encore faire ressortir les unités qui présentent des caractères communs au niveau de certaines variables. En d'autres mots la matrice ordonnable de Bertin permet, d'une part, de dégager les relations factorielles entre des groupes de variables, et d'autre part de régionaliser les différentes parties d'un territoire en fonction de la répartition des valeurs des variables étudiées.

Le regroupement optimum des lignes et des colonnes est obtenu lorsque l'image donnée par les parties foncées de la matrice (valeurs fortes) est la plus proche possible d'une diagonale (figure 11). Cette disposition permet de faire ressortir les oppositions entre les valeurs fortes et les valeurs faibles, pour un groupe de variables donné, ou pour un groupe d'unités géographiques: les nuances intermédiaires, c'est-à-dire les variations du gris, sont aussi ordonnables selon le même principe. Cette manipulation de la matrice par le jeu des permutations peut paraître très subjective à l'observateur non initié; avec un peu d'entraînement on s'aperçoit qu'elle permet de parvenir aux mêmes résultats (mais non chiffrés) qu'un calcul de corrélation multiple ou une analyse factorielle. Par rapport aux méthodes numériques, elle a l'avantage de permettre le test de toutes sortes d'hypothèses définies par le chercheur tout en laissant à celui-ci le plein contrôle de la manipulation des données durant toutes les phases du traitement, ce qui n'est pas le cas avec les méthodes statistiques, dépendantes par ailleurs d'une foule de conditions préalables. Enfin, un autre des avantages de la matrice ordonnable de Bertin est que le chercheur garde toujours l'ensemble de l'information sous les yeux. 


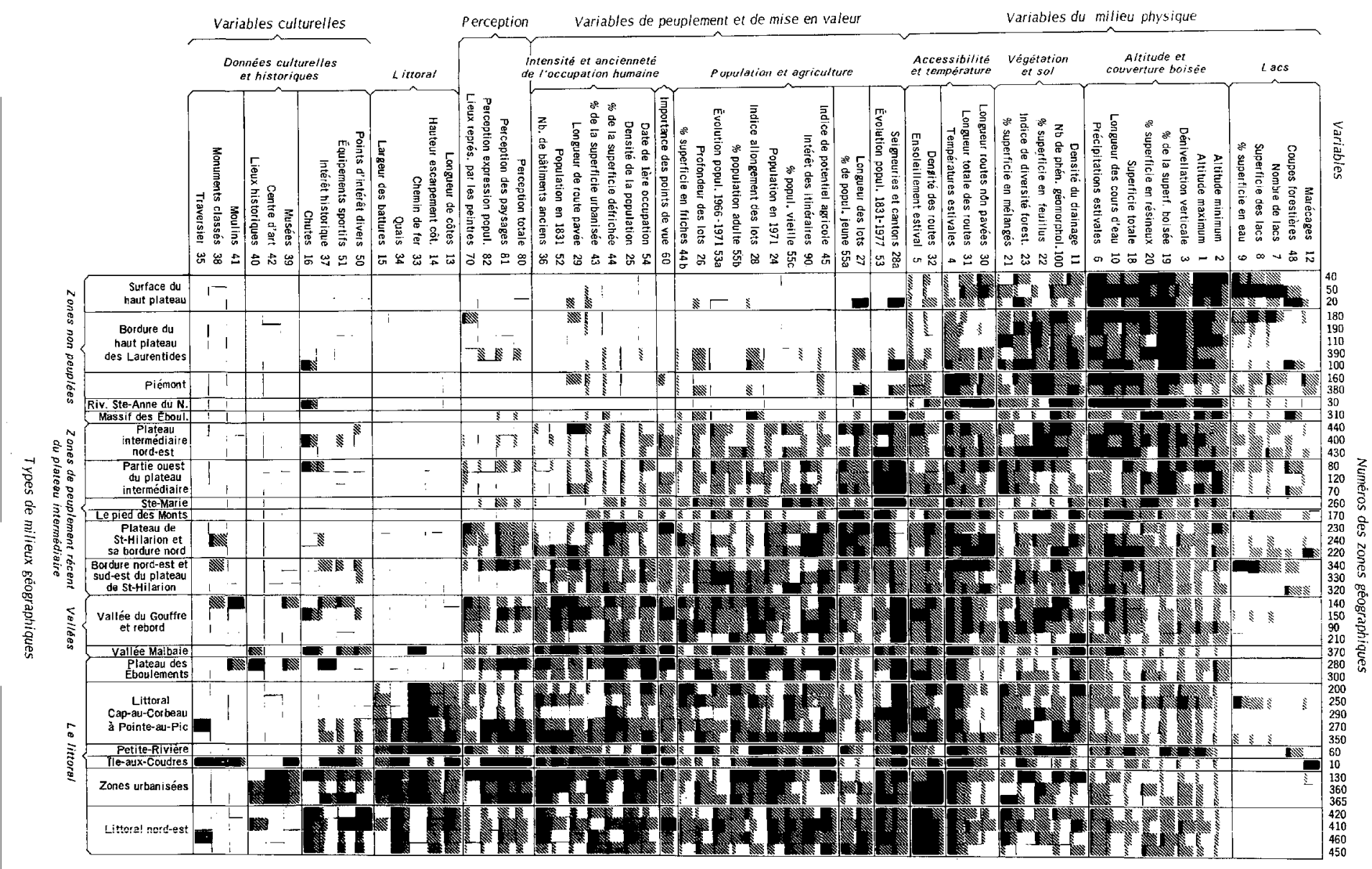

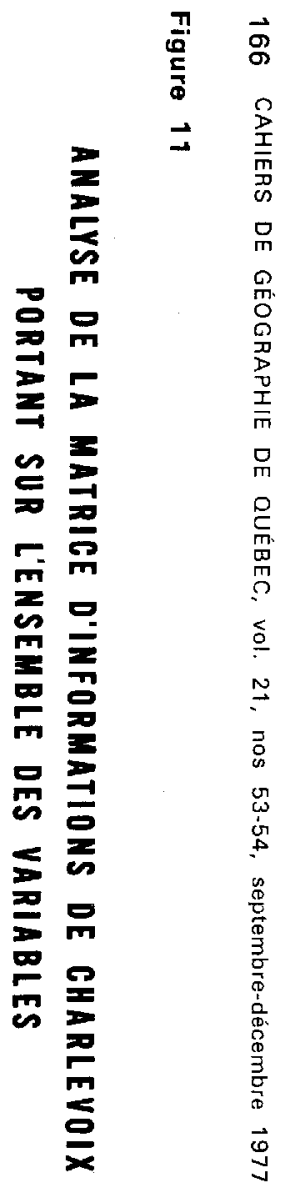


Examinons maintenant quels sont les résultats auxquels ont abouti le traitement de la matrice d'informations pour Charlevoix et l'analyse en composantes principales des mêmes informations.

\section{CLASSIFICATION DES PAYSAGES DE CHARLEVOIX ET ÉVALUATION DE LEUR "POTENTIEL CULTUREL »}

L'analyse des données de l'inventaire spatial de Charlevoix s'est effectuée en deux étapes: dans un premier temps nous avons défini les caractéristiques générales des différentes zones et dans un second temps nous avons tenté d'établir une hiérarchie des zones en fonction des variables pertinentes aux objectifs de délimitation des sites et arrondissements culturels. La première analyse ne préjuge pas de l'intérêt plus ou moins grand que peuvent présenter certaines variables et s'en tient à une stricte classification. La seconde, au contraire, introduit des jugements de valeur.

\section{Différenciation des zones à partir de l'analyse de l'ensemble des variables}

Nous avons utilisé simultanément l'analyse en composantes principales et celle de la matrice visuelle de Bertin afin de comparer les résultats respectifs. L'analyse en composantes principales a été appliquée à 42 des 72 variables originales ${ }^{10}$, plusieurs d'entre elles étaient en effet trop interdépendantes ou ne s'appliquaient qu'à un trop petit nombre de zones. Les coefficients de corrélation de Pearson ont été utilisés comme éléments de la matrice d'entrée de l'analyse en composantes principales. Sur la figure 12 on a illustré les coefficients de corrélation supérieurs à 0,60 et les groupements de variables qu'il est possible d'observer en fonction de ces coefficients. L'analyse comparée de la matrice visuelle de Bertin (figures 11 et 12 ) et de la matrice de corrélation révèle des groupements semblables de variables. En premier lieu on observe le groupe cohérent des variables relatives au relief et au climat, qui s'opposent aux variables marquant l'intensité de l'occupation humaine. Un second bloc identifie les traits de l'urbanisation, en opposition avec l'importance des superficies boisées. Les traits du cadastre sont étroitement liés entre eux et avec l'ancienneté du peuplement. Enfin, on remarque l'association entre les caractères liés à l'agriculture et certains éléments du patrimoine architectural.

L'analyse en composantes principales avec rotation "varimax » a permis de faire ressortir trois facteurs principaux expliquant respectivement $17,4 \%$, $14,4 \%$ et $13 \%$ de la variance (tableau 4 ), et qui étaient déjà visibles dans la matrice de corrélation. Le premier facteur met en évidence l'opposition entre deux pôles: un pôle positif marqué par l'altitude élevée, la forte pluviosité et la forêt de résineux; un pôle négatif illustrant l'ancienneté du peuplement, le cadastre et l'ensoleillement. Le second facteur regroupe des variables liées à l'urbanisation. Le troisième facteur souligne l'association entre l'agriculture et le patrimoine historique.

La classification issue de l'analyse en composantes principales par le programme dit des "h-groupes" et portant sur 11 facteurs, fait ressortir des similitudes avec le classement effectué sur la matrice visuelle. Le classement n'est toutefois pas comparable du fait que les deux analyses n'incorporent 
Figure 12

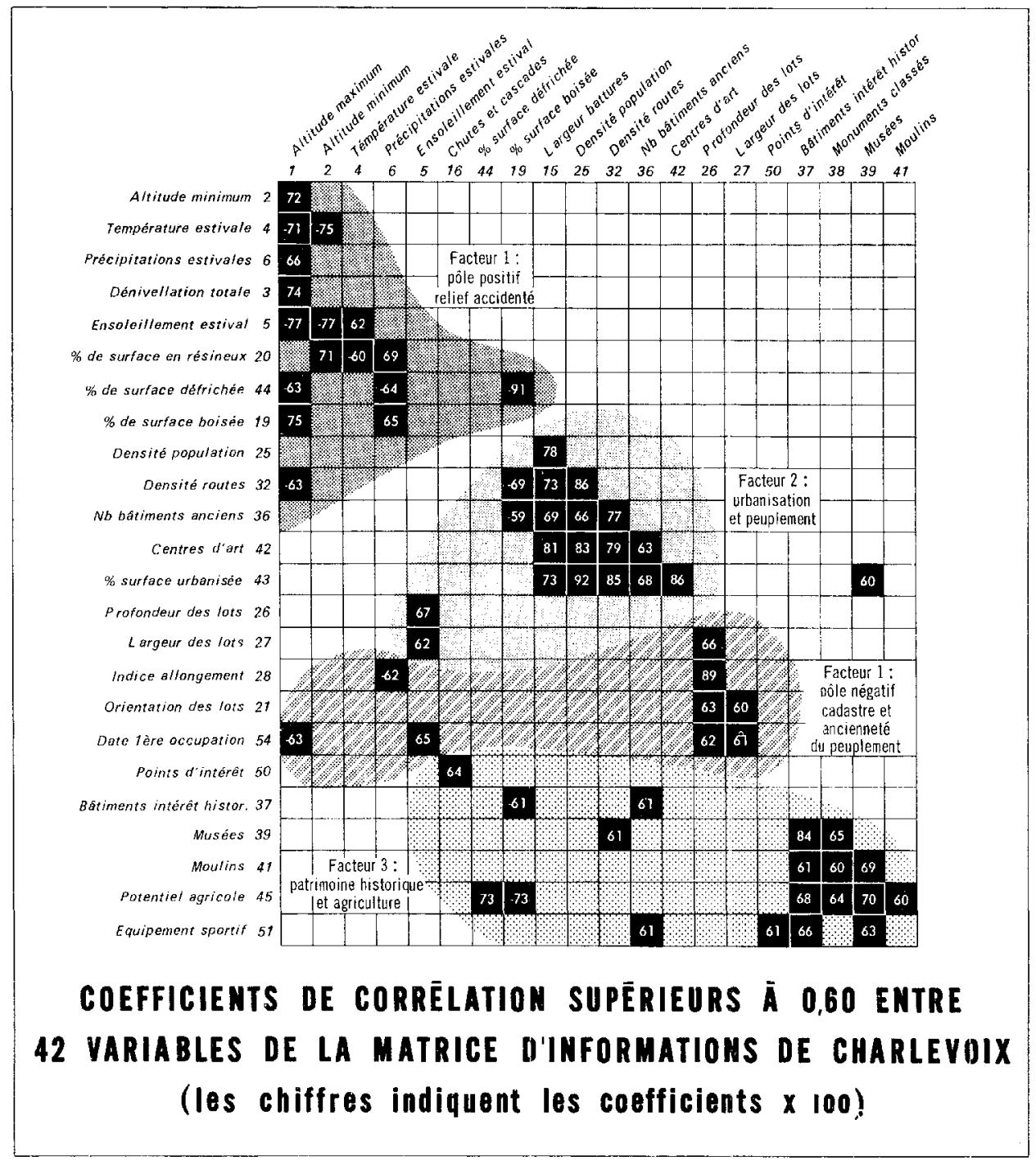

pas le même nombre de variables. Aussi avons-nous retenu seulement le classement à partir de la matrice visuelle, qui retient plus de variables. Tout en faisant ressortir les oppositions entre les groupes de variables définis précédemment, il ordonne les zones en tenant compte de leur situation et de leur contiguilté géographique, ainsi que des caractères différentiels du peuplement (figures 11 et 13). L'agencement spatial des 24 variables principales du milieu physique, des 17 variables de peuplement contemporain et des 19 variables aléatoires (milieu physique, peuplement, perception) conduit à l'identification de quatre grands groupes d'unités géographiques (figure 11 et tableau 5) : 


\section{Tableau 4}

\section{Composition des trois premiers facteurs issus de l'analyse en composantes principales}

\section{FACTEUR 1, expliquant $17,4 \%$ de la variance}

Pôle positif: Relief accidenté, pluie et boisés résineux variables :

saturation

Altitude maximum

0,69157

Altitude minimum

0,67261

Nombre de jours de pluie

0,54209

$\%$ de surface en eau

0,52173

$\%$ couvert par les boisés résineux

0,49678

Pôle négatif: Ancienneté du peuplement, cadastre, ensoleillement variables

saturation

Profondeur des lots

0,85558

Largeur des lots

0,82170

Ensoleillement relatif

0.81473

Orientation des lots

0,69482

Date de première occupation

0,69138

Indice d'allongement des lots

0,68707

FACTEUR 2, expliquant $14,3 \%$ de la variance

Pôle positif : Urbanisation et peuplement variables :

saturation

Centres d'art

0,94903

\% urbain et bâti

0,93563

Densité de population

0,93536

Densité des routes par $\mathrm{km}^{2}$

0.83117

Largeur des battures

0.82995

Nombre de bâtiments anciens

0,69442

Musées

0,54201

FACTEUR 3, expliquant $13,1 \%$ de la variance

Pôle positif: Agriculture et patrimoine historique variables

saturation

Monuments classés

0,83363

Potentiel agricole 3

0,82758

Moulins

0.82703

Musées

0,79285

Intérêt historique et autre

0.75623

Mines et gravières

0.64242

Équipements sportifs

0,57038

Pôle négatif: Régions boisées variable :

saturation

\% Couverture boisée

0,48118 
Figure 13

\section{SYNTHẼSE DES TYPES DE MILIEUX GËOGRAPHIQUES DE CHARLEVOIX}

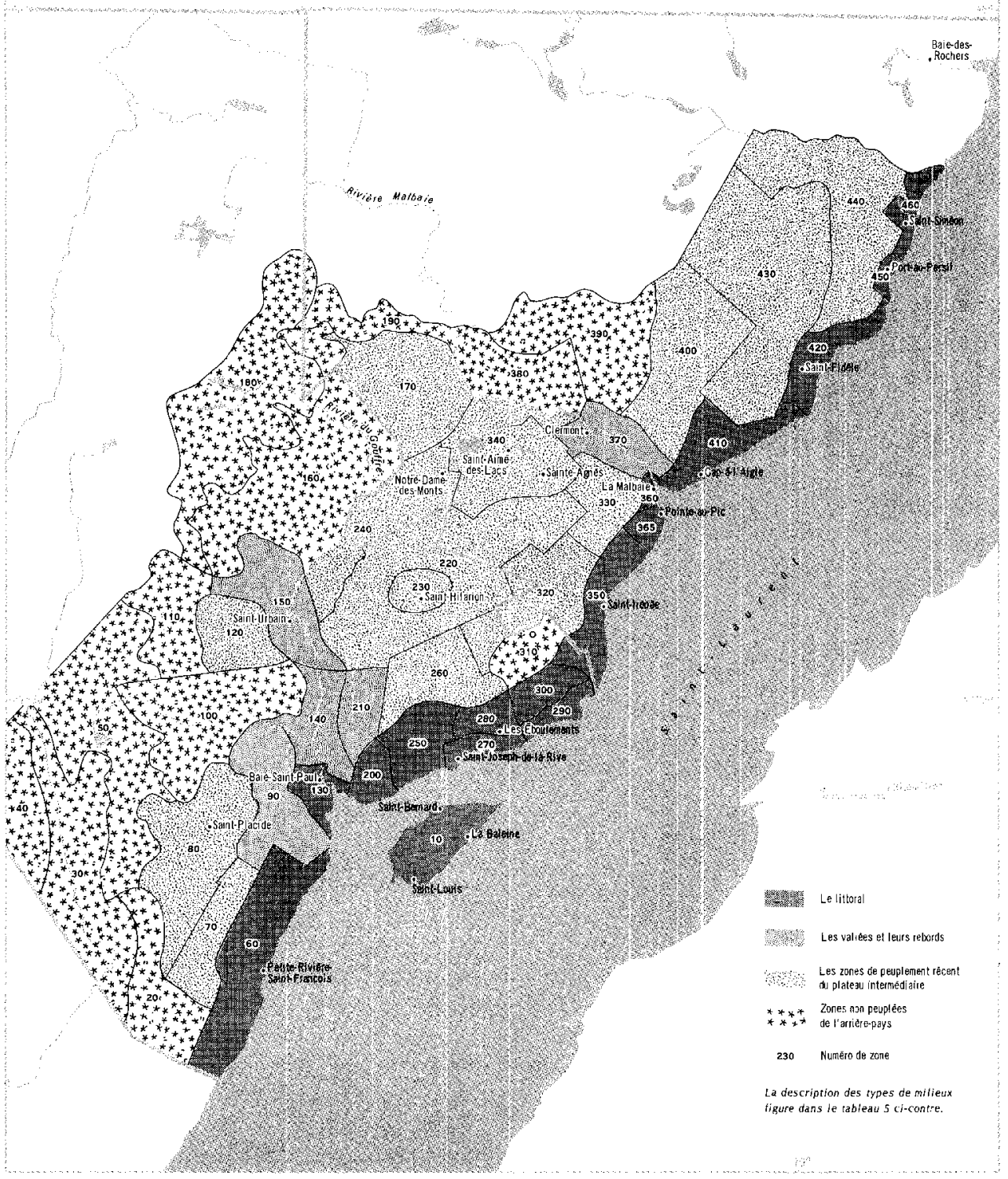


Tableau 5

Types de milieux géographiques dans Charlevoix

\author{
VARIABLES DOMINANTES \\ (pour le détail, voir figure 11)
}

\section{IDENTIFICATION DES ZONES CONSTITUANTES}

(la localisation des numéros de zones est indiquée sur les figures 7 et 13)

\section{Zones sans population}

Altitude élevée et fortes dénivellations : Abondance de couverture forestière avec dominante de résineux; abondance de l'hydrographie : extrêmes climatiques nombreux phénomènes géomorphologiques; faible accessibilité : absence de peuplement.
$20,40,50$ : Surface du haut plateau des Laurentides

100, 110, 180, 190, 390: bordure du haut plateau des Laurentides

160, 380 : piémont

30: vallée de la rivière Sainte-Anne

310: massif des Éboulements

\section{Zones de peuplement récent du plateau intermédiaire}

Altitude intermédiaire

$(150-600 \mathrm{~m})$; peuplement : seconde moitié de XIXe; faible densité de population :

différences internes basées sur la situation géographique, le cadastre, l'importance relative de l'agriculture, l'ancienneté du peuplement.
400, 430, 440: plateau intermédiaire, partie nord-est

$70,80,120$ : plateau intermédiaire, partie ouest

260: Ste-Marie de Charlevoix

170 : le pied des monts

$220,230,240$ : le plateau de Saint-

Hilarion et sa bordure nord

$320,330,340$ : bordure nord et sudorientale du plateau de Saint-Hilarion

Les vallées et leurs rebords (à l'exclusion des deux estuaires)

Présence des deux cours d'eau les plus importants ;

variété des formes géomorphologiques

et de la végétation ;

conditions climatiques plus favorables;

peuplement de régime français

(seigneuries) ou anglais :

paysages agraires bien développés:

peuplement relativement intense ;

vestiges architecturaux nombreux.
90, 140, 210, 150 : vallée du Gouffre 370: vallée de la rivière Malbaie

\section{Le littoral}

Intensité des traits de peuplement; variété de la végétation :

influences climatiques marines:

ancienneté du peuplement et seigneuries ; densité importante de population et urbanisation :

richesse du patrimoine architectural: nombreux éléments d'intérêt culturel et historique ;

relations entre le peuplement et le fleuve ; caractéristiques physiques liées au littoral.
280, 300 : plateau des Éboulements

200, 250, 270, 290, 350 : le littoral

du cap au Corbeau à Pointe-au-Pic

60: Petite-Rivière-Saint-François

10 : l'île aux Coudres

$130,360,365$ : les zones urbanisées Baie-Saint-Paul, La Malbaie, Pointe-au-Pic

$410,420,450,460$ : le littoral au nord-est de la Malbaie 
- les zones sans population, où les traits physiques identifiés dans le facteur 1 dominent ;

- les zones de peuplement récent du plateau intermédiaire caractérisées par un relief moins accidenté et une agriculture marginale ;

- les vallées et leurs rebords, où l'urbanisation, l'agrıculture et l'ancierıneté du peuplement dominent (facteurs 2 et 3 ) ;

- le littoral, dont la vigueur de l'escarpement et l'ancienneté du peuplement décroissent du sud-ouest au nord-est.

L'analyse en composantes principales et la matrice visuelle de Bertin permettent donc d'aboutir à des résultats complémentaires. L'approche mathématique fournit des résultats plus précis au niveau de l'établissement des relations entre les variables. Par contre, la matrice visuelle permet d'apporter des nuances dans la classification sans jamais perdre de vue les données originales: elle est beaucoup plus souple lors de la recherche d'ensembles régionaux homogènes car elle permet au chercheur de faire intervenir sa connaissance du terrain, ce qui est impossible avec l'analyse mathématique. Enfin, la possibilité de travailler sur une partie seulement de la matrice nous conduit à examiner de plus près les variables liées au "potentiel culturel".

\section{Classification des zones de Charlevoix en fonction de leur "potentiel} culturel "

Parmi les types de milieux géographiques différenciés lors du premier traitement des variables, il reste à établir une hiérarchie en fonction de critères qui pourraient servir éventuellement à la désignation de sites ou arrondissements culturels. Or, sur les quelques 70 variables disponibles, il est assez difficile de séparer celles qui ont ur caractère favorable ou défavorable à une telle désignation. Cette remarque est surtout valable pour les variables bio-physiques, puisque les différentes parties de Charlevoix présentent chacune des traits vigoureux et qu'on ne peut affirmer, a priori, que la côte est plus spectaculaire que l'intérieur montagneux, ou vice-versa. Éventuellement, on pourrait affirmer que certains caractères climatiques ont des incidences positives ou négatives sur l'attrait des différentes parties de la région. Mais, là encore, il n'y a rien d'absolu puisque la côte peut bénéficier d'un climat plus doux mais plus humide, alors que certaines parties de l'intérieur (Saint-Urbain) possèdent un climat plus froid mais plus sec et ensoleillé.

Par ailleurs, il est très difficile de définir un "potentiel " théorique pour un arrondissement culturel puisque les "fonctions" d'un tel arrondissement sont encore très peu explicitées dans les termes de la loi. On peut cependant tenter de proposer cles groupes de paramètres destinés à nous servir de guide: possibilités de découverte visuelle des paysages, degré d'humanisation, charge culturelle (éléments visibles et invisibles), infrastructures sociales et culturelles, perception des lieux par la population locale. C'est sur cette base que nous avons isolé certaines variables destinées à illustrer le «potentiel culturel» de Charlevoix (tableau 6). Parmi 
Tableau 6

Variables retenues pour identifier le "potentiel culturel " de Charlevoix

Le contact avec le Saint-Laurent

et les chutes

- longueur de côte

- hauteur de l'escarpement côtier

- largeur des battures

- quais

- traversier

- numbre de chutes

Possibilités de découverte visuelle des paysages

- points de vue

- itinéraires en surplomb
Paysages agraires

- seigneuries et cantons

- allongement des lots

- potentiel agricole

- importance de la superficie défrichée

\author{
Infrastructure socio-culturelle \\ - points d'intérêt socio-culturels \\ - équipements sportifs \\ - musées \\ - centres d'art
}

\section{Perception des paysages}

- lieux représentés par les peintres

- perception globale par la population locale

- perception des paysages

- expression populaire

- bâtiments anciens

- monuments classés

- moulins

les variables proposées on remarquera la faible importance des variables bio-physiques. Ceci est d'abord dû à la difficulté de leur attribuer une signification "positive " ou "négative " en vue de la classification des sites. Par ailleurs, leur incidence culturelle n'a pas encore été précisée par des études de perception spécifiques. Elles sont de toute manière incorporées dans les variables "points de vue " et "itinéraires".

Pour chaque variable sélectionnée, on a attribué les cotes $6,5,4$, 3, 2, 1 aux six niveaux définis lors du montage de la matrice d'informations. Ensuite, dans chacune des zones d'analyse, on a effectué la simple sommation des cotes de chacune des variables présentes. Nous n'avons pas tenté d'établir une pondération quelconque étant donné l'absence de critères sûrs qui en garantiraient l'objectivité. La présence d'une trentaine de variables pertinentes assure cependant une certaine validité aux résultats. Le classement des zones de Charlevoix selon leur potentiel culturel figure dans le tableau 7 . 
Tableau 7

\section{Classement des zones de Charlevoix selon les variables indicatrices du "potentiel culturel"}

\begin{tabular}{|c|c|c|c|c|c|}
\hline & $\begin{array}{c}\text { Milieu } \\
\text { géographique }\end{array}$ & $\begin{array}{l}\text { Numéro } \\
\text { de zone }\end{array}$ & Lieu & $\begin{array}{l}\text { Pointage } \\
\text { sans la } \\
\text { perception }\end{array}$ & $\begin{array}{l}\text { Pointage } \\
\text { avec } \\
\text { perception }\end{array}$ \\
\hline A. & $\begin{array}{l}\text { Berceau de } \\
\text { Charlevoix }\end{array}$ & $\begin{array}{r}10 \\
130\end{array}$ & $\begin{array}{l}\text { Île aux Coudres } \\
\text { Baie-Saint-Paul }\end{array}$ & $\begin{array}{r}109 \\
98\end{array}$ & $\begin{array}{l}127 \\
116\end{array}$ \\
\hline B. & $\begin{array}{l}\text { Littoral en } \\
\text { amont du } \\
\text { cap à l'Aigle }\end{array}$ & $\begin{array}{r}410 \\
280 \\
350 \\
360 \\
270 \\
365 \\
60\end{array}$ & $\begin{array}{l}\text { Cap-à-I'Aigle } \\
\text { Les Éboulements } \\
\text { Saint-Irénée } \\
\text { La Malbaie } \\
\text { Saint-Joseph-de-la-Rive } \\
\text { Pointe-au-Pic } \\
\text { Petite-Rivière-Saint-François }\end{array}$ & $\begin{array}{l}82 \\
74 \\
71 \\
71 \\
67 \\
64 \\
67\end{array}$ & $\begin{array}{l}92 \\
89 \\
87 \\
84 \\
83 \\
81 \\
74\end{array}$ \\
\hline c. & $\begin{array}{l}\text { Les grandes } \\
\text { vallées }\end{array}$ & $\begin{array}{l}140 \\
370 \\
150\end{array}$ & $\begin{array}{l}\text { Rivière-du-Gouffre } \\
\text { Rivière-Malbaie-Clermont } \\
\text { Saint-Urbain }\end{array}$ & $\begin{array}{l}69 \\
62 \\
53\end{array}$ & $\begin{array}{l}77 \\
71 \\
65\end{array}$ \\
\hline D. & $\begin{array}{l}\text { Le littoral } \\
\text { nord-est }\end{array}$ & $\begin{array}{l}420 \\
460 \\
450\end{array}$ & $\begin{array}{l}\text { Saint-Fidèle } \\
\text { Saint-Siméon } \\
\text { Port-au-Persil }\end{array}$ & $\begin{array}{l}55 \\
54 \\
51\end{array}$ & $\begin{array}{l}62 \\
64 \\
57\end{array}$ \\
\hline E. & $\begin{array}{l}\text { Zones de } \\
\text { transition, } \\
\text { "portes " et } \\
\text { aproches }\end{array}$ & $\begin{array}{l}90 \\
200 \\
250 \\
290 \\
300\end{array}$ & $\begin{array}{l}\text { Terrasses Ouest de Baie- } \\
\text { Saint-Paul (rang St-Antoine) } \\
\text { Cap au Corbeau } \\
\text { Misère } \\
\text { Cap aux Oies } \\
\text { Les Éboulements } \\
\text { Les Éboulements-Est }\end{array}$ & $\begin{array}{l}45 \\
45 \\
47 \\
42 \\
38\end{array}$ & $\begin{array}{l}51 \\
51 \\
50 \\
47 \\
41\end{array}$ \\
\hline F. & $\begin{array}{l}\text { Rebord nord } \\
\text { du plateau }\end{array}$ & $\begin{array}{l}340 \\
240\end{array}$ & $\begin{array}{l}\text { Sainte-Agnès - } \\
\text { Saint-Aimé-des-Lacs } \\
\text { Notre-Dame-des-Monts - } \\
\text { Rg Saint-Jean-Baptiste }\end{array}$ & $\begin{array}{l}40 \\
35\end{array}$ & $\begin{array}{l}5.3 \\
45\end{array}$ \\
\hline G. & $\begin{array}{l}\text { Plateau de } \\
\text { Saint-Hilarion }\end{array}$ & $\begin{array}{l}230 \\
220\end{array}$ & $\begin{array}{l}\text { Saint-Hilarion-village } \\
\text { Saint-Hilarion-campagne }\end{array}$ & $\begin{array}{l}31 \\
32\end{array}$ & $\begin{array}{l}40 \\
39\end{array}$ \\
\hline $\mathrm{H}$. & $\begin{array}{l}\text { "Poches " du } \\
\text { plateau }\end{array}$ & $\begin{array}{l}330 \\
320 \\
260 \\
210\end{array}$ & $\begin{array}{l}\text { Rivière Mailloux (La Malbaie) } \\
\text { Rochette (Saint-Irénée) } \\
\text { Sainte-Marie } \\
\text { (Les Éboulements) } \\
\text { Rang Saint-Ours } \\
\text { (Rivière-du-Gouffre) }\end{array}$ & $\begin{array}{l}35 \\
29 \\
28 \\
32\end{array}$ & $\begin{array}{l}35 \\
29 \\
34 \\
32\end{array}$ \\
\hline I. & $\begin{array}{l}\text { Vallées } \\
\text { transversales }\end{array}$ & $\begin{array}{r}80 \\
400\end{array}$ & $\begin{array}{l}\text { Saint-Placide (Baie-Saint-Paul) } \\
\text { Grand-Fonds (Rivière-Malbaie) }\end{array}$ & $\begin{array}{l}30 \\
28\end{array}$ & $\begin{array}{l}36 \\
30\end{array}$ \\
\hline J. & $\begin{array}{l}\text { Gradins } \\
\text { occidentaux }\end{array}$ & $\begin{array}{r}120 \\
70 \\
100\end{array}$ & $\begin{array}{l}\text { Rang Saint-Jérôme } \\
\text { (Saint-Urbain) } \\
\text { Saint-Cassien (Petite-Rivière) } \\
\text { Haute Rivière de la Mare }\end{array}$ & $\begin{array}{l}21 \\
20 \\
17\end{array}$ & \\
\hline K. & $\begin{array}{l}\text { Massif des } \\
\text { Éboulements }\end{array}$ & 310 & Massif des Éboulements & 17 & \\
\hline
\end{tabular}


Tableau 7 (suite)

Classement des zones de Charlevoix selon les variables indicatrices du "potentiel culturel "

\begin{tabular}{|c|c|c|c|c|c|}
\hline & $\begin{array}{l}\text { Milieu } \\
\text { géographique }\end{array}$ & $\begin{array}{l}\text { Numéro } \\
\text { de zone }\end{array}$ & Lieu & $\begin{array}{c}\text { Pointage } \\
\text { sans la } \\
\text { perception }\end{array}$ & $\begin{array}{c}\text { Pointage } \\
\text { avec } \\
\text { perception }\end{array}$ \\
\hline L. & $\begin{array}{l}\text { Plateau } \\
\text { Nord-Est }\end{array}$ & $\begin{array}{l}440 \\
430\end{array}$ & $\begin{array}{l}\text { Rivière Noire (Saint-Siméon) } \\
\text { Arrière de Saint-Fidèle }\end{array}$ & $\begin{array}{l}17 \\
17\end{array}$ & \\
\hline M. & $\begin{array}{l}\text { Gradins } \\
\text { nord }\end{array}$ & $\begin{array}{l}390 \\
380 \\
170 \\
160\end{array}$ & $\begin{array}{l}\text { Rivière Jacob (Clermont, } \\
\text { Rivière Malbaie) } \\
\text { Rivière Signole (Clermont) } \\
\text { Canton de Sales (St-Aimé, } \\
\text { N.-D.-des-Monts) } \\
\text { Lac des Cygnes, Lac Ontario } \\
\text { (St-Urbain) }\end{array}$ & $\begin{array}{r}15 \\
9 \\
10 \\
5\end{array}$ & \\
\hline N. & $\begin{array}{l}\text { Plateau } \\
\text { sud-ouest }\end{array}$ & $\begin{array}{l}20 \\
30\end{array}$ & $\begin{array}{l}\text { Lac La Flippe } \\
\text { Rivière Ste-Anne du Nord }\end{array}$ & $\begin{array}{l}7 \\
5\end{array}$ & \\
\hline 0. & $\begin{array}{l}\text { Plateau } \\
\text { ouest }\end{array}$ & $\begin{array}{r}40 \\
50 \\
110\end{array}$ & $\begin{array}{l}\text { Lac Louis } \\
\text { Lac Ripault } \\
\text { Gros Bras }\end{array}$ & $\begin{array}{l}0 \\
0 \\
0\end{array}$ & \\
\hline P. & $\begin{array}{l}\text { Haute } \\
\text { Montagne }\end{array}$ & $\begin{array}{l}180 \\
190\end{array}$ & $\begin{array}{l}\text { Chaîne des Monts } \\
\text { Canton Lacoste }\end{array}$ & $\begin{array}{l}0 \\
0\end{array}$ & \\
\hline Q. & $\begin{array}{l}\text { Haute } \\
\text { Montagne }\end{array}$ & & Haute rivière Malbaie & $\begin{array}{l}\text { Parties non } \\
\text { dans la } \\
\text { d'informati }\end{array}$ & $\begin{array}{l}\text { incorporées } \\
\text { trice } \\
\text { on }\end{array}$ \\
\hline R. & $\begin{array}{l}\text { Littoral } \\
\text { sauvage }\end{array}$ & & Baie des Rochers & & \\
\hline
\end{tabular}

Comparaison du classement obtenu par les études de perception avec celui résultant du traitement de 27 variables (tableau 8)

Le classement relatif des localités ou municipalités de Charlevoix découlant des enquêtes de perception menées auprès de la population locale présente des similitudes mais aussi des différences notables avec le classement obtenu précédemment à partir de 27 variables. La comparaison des deux classements (tableau 8) permet de faire ressortir de manière très nette, dans le traitement des variables, la surévaluation systématique des zones littorales et la sous-évaluation de celles situées à l'intérieur. Ceci impliquerait probablement une pondération plus faible des variables liées au littoral et l'introduction de quelques variables mettant en valeur les caractéristiques propres à l'intérieur.

Dans le détail, on peut noter que la zone de Saint-Hilarion est la plus défavorisée par les variables. La bordure nord du plateau l'est également à un degré notable, ainsi que Saint-Urbain. La surévaluation relative de Pointe-au-Pic par la perception peut être due en partie au fait que le 
Tableau 8

Classement mixte des localités ou municipalités de Charlevoix (perception et 27 variables)

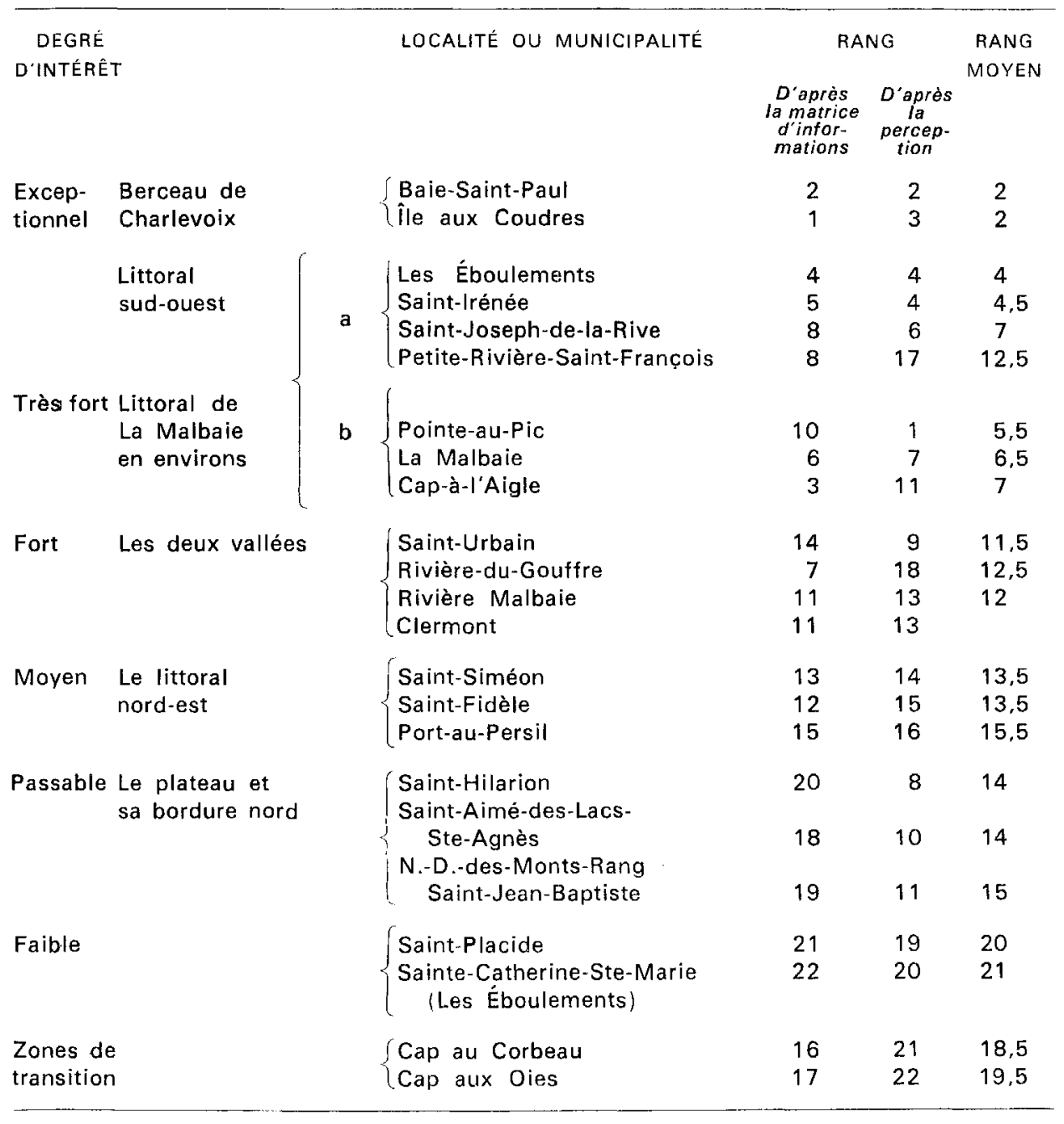

manoir Richelieu, noté séparément lors des enquêtes, a été intégré à Pointe-au-Pic dans le classement, d'où une certaine redondance qui favorise Pointe-au-Pic. La partie inférieure de la vallée du Gouffre (municipalité de Rivière-du-Gouffre) a probablement été souvent confondue avec Baie-StPaul lors des enquêtes de perception, ce qui explique son faible pointage sous cette rubrique. Petite-Rivière-Saint-François et Cap-à-l'Aigle sont en bonne position dans le classement des variables, alors qu'elles ressortent peu avec la perception. Il faut $y$ voir l'effet positif des variables littorales et la sous-estimation des valeurs historiques et culturelles par la perception. Dans le cas de Petite-Rivière, on pourrait dire également que sa situation en cul-de-sac ne la favorise guère dans les circuits projetés, du fait qu'il faut emprunter le même chemin à l'aller et au retour. Enfin, cap aux Oies et cap au Corbeau sont favorisés par les variables littorales. 
Le couple Baie-Saint-Paul - île aux Coudres se dégage incontestablement dans les deux cas. Dans le second bloc, Les Éboulements, SaintIrénée, La Malbaie et Saint-Joseph-de-la-Rive ne bougent guère d'un classement à l'autre. Pointe-au-Pic et Cap-à-l'Aigle suscitent des divergences mais géographiquement elles s'intègrent parfaitement à la continuité du peuplement littoral. Le troisième bloc identifie les deux vallées principales, dont le potentiel culturel peut prêter à controverse. Petite-RivièreSaint-François, sous-estimée par la perception, mériterait certainement d'être replacée dans la continuité du second bloc (le littoral). Le groupe Saint-Siméon, Saint-Fidèle et Port-au-Persil correspond aux poches de peuplement du littoral nord-est. Le plateau de Saint-Hilarion et sa bordure nord constituent le cinquième groupe dont les paysages et les caractéristiques du peuplement sont bien spécifiques. Les deux caps arrivent en sixième position alors que les enclaves de Saint-Placide et Sainte-Catherine prennent la dernière place. Ce classement synthétique nuancé par les caractéristiques détaillées fournies par la matrice d'informations, ainsi que par les éléments de dégradation des paysages étudiés ci-après, constitue un cadre de référence pour la formulation de recommandations adéquates concernant le classement de sites et arrondissements naturels et d'une façon générale sur la conservation et la mise en valeur du patrimoine culturel de Charlevoix.

\section{DE LA CLASSIFICATION À LA PROTECTION ET $\grave{A}$ LA MISE EN VALEUR DES PAYSAGES}

Lors de l'évaluation du "potentiel culturel " des différentes zones de Charlevoix, nous n'avons considéré que la présence ou l'absence d'éléments positifs pour l'obtention d'un tel potentiel. Mais il existe aussi des facteurs négatifs de pollution physique et visuelle du milieu qui peuvent venir contrebalancer, et parfois même détruire, l'attrait des paysages les plus prometteurs. Les différentes formes de pollution sont liées à l'exploitation plus ou moins intensive, et parfois incontrôlée, des ressources présentes dans certaines parties du territoire. Leur identification est un préalable à toute action de protection et de mise en valeur.

\section{Formes de pollution des paysages}

\section{Pollutions liées à l'exploitation du milieu naturel}

L'exploitation du bois et en particulier la coupe à blanc de vastes étendues de forêts, notamment en bordure des lacs et des courś d'eau, engendre des cicatrices qui resteront présentes durant des dizaines d'années. De telles coupes ne sont guère visibles à l'intérieur du territoire municipalisé de Charlevoix. Par contre, les sites pittoresques de la haute rivière Malbaie sont menacés par ces coupes, la rivière elle-même étant dotée de plusieurs barrages servant à contrôler le flottage du bois.

Dans le domaine de l'exploitation agricole, on peut identifier des types de dégradation visuelle liés à l'envahissement des friches, ce dernier étant souligné par la présence de bâtiments en ruines. En 1966 la friche occupait déjà de 15 à $30 \%$ du territoire dans les zones agricoles de Charlevoix 
(relevé ARDA). Ce sont les aproches de Baie-Saint-Paul et des Éboulements qui sont les plus affectées par les friches; mais on les trouve aussi en abondance dans des zones densément peuplées : vallée du Gouffre, périphérie des Éboulements et de Cap-à-l'Aigle, île aux Coudres. La protection de l'intérêt esthétique des paysages agraires appelle plusieurs mesures correctives, dont le reboisement planifié des sols les plus ingrats et un encouragement à la mise en valeur des bons sols dans les secteurs définis comme étant viables économiquement ou dépendants de la fréquentation touristique. On peut mentionner aussi certains types de productions sans sol, tels les poulaillers et porcheries, dont l'aspect visuel ou l'odeur n'ont rien d'attirant ; mais pour l'instant ces productions restent confinées hors des circuits fréquentés (hameau de Rochette à Saint-Irénée).

Pollutions liées à l'urbanisation

L'extension anarchique du développement domiciliaire de type urbain constitue la menace la plus sérieuse pour la conservation des paysages ruraux. Dans les deux vallées principales, ce développement se traduit par la densification linéaire de I'habitat, encerclant et étouffant progressivement les espaces agricoles. La vallée du Gouffre, en amont et en aval de BaieSaint-Paul, possède encore des paysages agraires bien entretenus. Cependant le lotissement linéaire se développe de manière insidieuse et, s'il n'est pas contenu dans des limites précises, il risque de détruire irrémédiablement la trame agraire qui constitue le charme principal de la vallée du Gouffe. Dans la vallée de la rivière Malbaie, entre La Malbaie et Clermont, les ravages d'une urbanisation anarchique ont déjà atteint des proportions irrémédiables. L'étroitesse du site, traversé par une route fréquentée (route 138) explique sans doute en partie l'enchevêtrement de commerces, d'industries, de dépotoirs et de résidences qui défigure les berges de la rivière.

La dégradation de l'habitat se limite heureusement à des bâtiments isolés dans la plupart des cas; ils sont d'autant moins visibles que le paysage environnant est plus ample. Les deux seules concentrations notables s'observent dans le rang Saint-Jérôme, à Saint-Urbain, et dans le hameau de Rivière-Mailloux, à l'ouest de la Malbaie. La présence d'éléments architecturaux discordants par rapport à l'environnement reste ponctuelle et ne constitue pas encore une menace sérieuse pour les paysages.

Certaines manifestations des activités commerciales sont en complète disharmonie avec le paysage environnant, notamment le long de la route 138 , aux sorties nord de Baie-Saint-Paul et La Malbaie : constructions hétéroclites, couleurs horripilantes, affichage disparate et envahissant, etc. À noter également les réservoirs à essence trop voyants situés à Cap-à-l'Aigle et SaintBernard. Les dépotoirs et cimetières de voitures ne se remarquent guère pour le moment car ils sont bien cachés par la végétation.

\section{Pollutions liées à l'industrie}

La seule industrie importante de Charlevoix est la Cie Donohue à Clermont qui produit du papier journal. Outre l'émanation des fumées dans l'air, cette usine pollue la rivière Malbaie par le flottage (en amont), les écorces, les eaux blanches et les lubrifiants jetés dans la rivière (Lavigne, 1971). Étant donné le caractère vital de cette industrie pour la région, le problème est de garder le niveau de pollution acceptable pour les 8000 
personnes qui vivent dans la vallée. L'industrie de la construction lédifices et routes) se manifeste sur tout le territoire par l'exploitation de nombreuses gravières qui produisent autant de plaies visuelles dans le paysage. Elles sont souvent localisées en bordure des routes, et parfois dans des sites panoramiques: on en trouve d'importantes sur les terrasses à l'ouest de BaieSaint-Paul ainsi qu'au sommet du cap au Corbeau. Sans réaménagement de terrain leur trace risque de demeurer longtemps dans le paysage puisque la végétation $\mathrm{y}$ reprend très mal.

\section{Pollutions liées aux voies de communications}

Les travaux de terrassement en bordure des routes ont pour effet de dénuder le sol qui, dans les matériaux meubles, se trouve rapidement raviné par l'érosion si le gazonnement n'est pas effectué aussitôt. II en résulte un effet de désolation des plus inesthétique. Les nouveaux tracés routiers peuvent aussi affecter irrémédiablement la qualité des paysages. Ainsi, à la Malbaie, la construction du boulevard à voies séparées en bordure du Saint-Laurent a détruit le contact naturel entre la terre et l'eau, tant sur le plan visuel que biologique. Ce boulevard a également coupé la population de son accès naturel au fleuve, la privant d'un lieu de détente et de contact avec la nature.

Les lignes de transmission hydro-électriques constituent la forme de pollution visuelle la plus frappante dans le paysage. En hiver, la neige souligne encore plus l'absence de végétation qui les accompagne. Deux lignes majeures traversent le territoire du nord-est au sud-ouest et recoupent toutes les grandes unités du paysage, à l'exception du fleuve.

Le délabrement et la désaffection des quais sont liés à la disparition de l'activité maritime locale. Leur remise en état se justifierait cependant en fonction du tourisme, pour la navigation de plaisance, pour les points de vue remarquables qu'ils offrent, et aussi pour la fonction sociale qu'ils jouent comme lieu de rencontre et d'échanges sociaux pour la population locale et les visiteurs.

\section{Risques de pollution liés à l'industrie touristique}

Le développement trop intensif de l'industrie touristique dans une région peut entraîner une dégradation des paysages, d'une part par la prolifération des commerces, services et équipements de loisirs qui s'installent sans aucun plan d'ensemble et d'autre part par la pression trop intense de la foule des touristes sur le milieu naturel environnant. Dans Charlevoix, l'industrie touristique est encore insuffisamment développée pour constituer une sérieuse menace à l'environnement. Tout au plus peut-on s'inquiéter des répercussions qu'entraînera l'aménagement d'une station de ski d'envergure internationale à Petite-Rivière-Saint-François. Le caractère homogène de I'habitat du village et l'étroitesse de son site littoral, commandent une grande prudence dans le développement de constructions qui devront s'intégrer aux caractéristiques uniques du site.

Enfin, on doit mentionner les transformations des comportements et des mentalités provoqués par l'afflux touristique dans un territoire donné. C'est le cas de l'île aux Coudres dont le genre de vie traditionnel a été popularisé par les films de Pierre Perrault. D'une part le visiteur est souvent 
déçu de ne pas retrouver dans l'île ce qu'il a vu dans les films, et d'autre part les insulaires ont évolué dans leur mode de vie et leurs mentalités. Il peut en résulter des frictions quand l'optique des deux groupes est par trop différente.

II reste maintenant à intégrer les observations concernant la pollution des paysages à celles découlant de leur classification selon le potentiel culturel. On peut en déduire des recommandations relatives à la protection des paysages.

\section{La protection des paysages}

En plaçant sur une même carte les renseignements relatifs à la classification des paysages et aux dangers de pollution qui les menacent (figure 14) on peut en extraire des indications utiles pour leur protection. II ne fait pas de doute que, dans Charlevoix, les paysages les plus spectaculaires, les plus diversifiés et les plus humanisés sont localisés sur le littoral et dans les deux vallées principales. Les limites visuelles de ces entités tracent une frontière à l'intérieur de laquelle on retrouve l'essentiel des paysages de Charlevoix les plus intéressants sur le plan culturel. A l'intérieur de ces limites on peut distinguer trois sous-ensembles dont les caractéristiques impliquent des types d'actions différents :

- L'arc Petite-Rivière, Baie-Saint-Paul, Saint-lrénée, incluant l'île aux Coudres. C'est la partie du littoral la plus chargée à la fois quant à la vigueur du contact plateau-fleuve et plateau-vallée, quant à l'ancienneté du peuplement, qui remonte au régime français, quant aux formes architecturales, à la prédominance des formes agraires, aux traditions matérielles et orales, etc. Si l'on excepte la ville de Baie-Saint-Paul, cette partie du littoral a relativement peu souffert des assauts de l'urbanisation et garde l'image d'une insertion harmonieuse de l'occupation humaine dans un paysage spectaculaire.

- Le secteur Pointe-au-Pic, La Malbaie, Cap-à-l'Aigle, auquel on peut greffer la basse vallée de la rivière Malbaie, est au contraire marqué par une forte densité d'occupation de l'espace et une urbanisation qui passe de la villégiature de luxe (Pointe-au-Pic) au développement péri-urbain anarchique (berges de la rivière Malbaie) par une zone de transition (La Malbaie) qui a été aménagée tant bien que mal malgré l'étroitesse du site. Seul le village de Cap-à-l'Aigle a conservé presque intact son cachet de villégiature rurale. Bien que dans ce secteur le patrimoine culturel soit lui aussi bien développé, le caractère urbain et industriel du milieu commande des types d'actions de sauvegarde et de mise en valeur différents de ceux que l'on pourra mettre en application dans l'arc situé au sud-ouest.

- Le littoral nord-est est caractérisé par des petites poches de peuplement isolées dont le caractère intime doit être préservé, mais qui ne se prêtent pas à une mise en valeur culturelle d'envergure.

Dans le détail, les types d'espace suivants méritent une protection particulière :

1) Paysages à caractère naturel

- Les berges des rivières Malbaie et du Gouffre

- Les sections accessibles du littoral 
- L'embouchure des deux grandes vallées

- Certaines échancrures du littoral : Port-au-Saumon, Port-au-Persil, Baiedes-Rochers

- L'escarpement littoral de Petite-Rivière-Saint-François

- Le mont des Éboulements

- Les gorges de la haute rivière Malbaie et le plateau environnant

- La vallée de la rivière Sainte-Anne-du-Nord.

\section{2) Les paysages agraires}

Dans le contexte québécois, les sols de Charlevoix ne sont pas particulièrement favorables au maintien d'une agriculture rentable, cependant, il est important de garder en culture les espaces actuellement exploités à des fins agricoles, à la fois pour maintenir une certaine auto-suffisance à l'échelle locale et pour conserver l'attrait des paysages humanisés de Charlevoix, notamment autour des villages et au fond des vallées. La carte indique les endroits où le maintien des paysages agraires s'avère essentiel.

\section{3) Les formes construites}

Même si Charlevoix n'est pas une région de monuments historiques remarquables, il existe quand même certaines caractéristiques d'une architecture régionale à préserver, au niveau des formes architecturales, de leur assemblage, du site, etc. Les agglomérations considérées comme ayant un intérêt majeur sont celles qui, tout en ayant conservé un lot important de bâtiments anciens (antérieurs à 1900), possèdent un site remarquable et n'ont guère été touchées par l'urbanisation contemporaine. On peut inclure Baie-Saint-Paul, Les Éboulements, Saint-Joseph-de-la-Rive, Saint-Louis-del'île-aux-Coudres, Saint-Irénée, Cap-à-I'Aigle. D'autres villages possèdent un stock de bâtiment moins anciens mais constituant cependant un ensemble assez homogène: Petite-Rivière, Saint-Urbain, Saint-Fidèle, Saint-Siméon. La zone Pointe-au-Pic-La Malbaie, bien que possédant des édifices remarquables, a été passablement transformée par l'urbanisation récente. À conserver également, les derniers vestiges de bâtiments agricoles construits selon le style régional de Charlevoix.

4) Itinéraire de liaison

La protection des axes routiers reliant les paysages remarquables de Charlevoix est le quatrième élément à citer : protection des zones de points de vue et protection visuelle des abords des itinéraires panoramiques. Il s'agit essentiellement des routes $138,362,381,170$, de la route de ceinture de l'île aux Coudres, de la route reliant Saint-Urbain à Notre-Dame-des-Monts, et du chemin d'accès aux gorges de la rivière Malbaie.

\section{Des arrondissements culture/s ou un développement culturel global ? 12}

En regardant la carte on s'aperçoit que c'est tout le territoire habité de Charlevoix qui mériterait d'être classé, à un titre ou à un autre, selon les termes de la Loi 2. En se restreignant, on pourrait proposer le classement du territoire correspondant en gros à la zone de peuplement du régime français: Petite-Rivière, Baie-Saint-Paul et la basse vallée du Gouffre, Les Éboulements, Saint-Irénée, Saint-Joseph-de-la-Rive, l'île aux Coudres. Comme on l'a dit précédemment, c'est là que l'on retrouve les paysages les plus 
spectaculaires, les formes d'occupation humaine et le patrimoine les plus riches et les plus authentiques de tout le territoire. Une telle délimitation d'arrondissement culturel serait certainement justifiée et fort défendable. Mais qu'adviendrait-il du reste du territoire? Le laisserait-on s'enlaidir? Le priverait-on de possibilités de développement culturel ?

Or il nous apparaît peu souhaitable, sinon préjudiciable pour la population locale, de créer un ou des arrondissements culturels qui deviendraient des réserves culturelles ou des sortes de ghettos où l'on donnerait les derniers vestiges du patrimoine de Charlevoix en pâture aux touristes. Au lieu de cela, il ne serait pas plus dispendieux de concevoir un plan de développement culturel global de toutes les parties de la région, selon la vocation de chacune, et selon différents niveaux d'intensité.

On s'aperçoit donc rapidement qu'il est indispensable de concevoir un plan de protection et de mise en valeur des paysages qui touche l'ensemble du territoire de Charlevoix et qui fasse appel non seulement aux moyens d'action gouvernementaux mais également à l'initiative de la population locale. La protection et la mise en valeur des paysages et du patrimoine culturel de Charlevoix devraient se faire essentiellement pour et par la population locale par le biais d'organismes populaires, avec une certaine aide technique des gouvernements, mais sans contrôle technocratique exagéré. Les opérations de mise en valeur reposent sur plusieurs types d'actions allant de l'échelle locale à l'échelle régionale, et dont la mise en oeuvre implique une intervention de la population locale à la base et de certaines instances gouvernementales au sommet. On peut ainsi distinguer des actions de sensibilisation, des actions restrictives, des actions correctives, des actions de développement culturel ponctuel et des actions de développement culturel régional. Les actions de sensibilisation font surtout appel au travail de la population de chaque localité, dans le cadre des groupements culturels locaux existants ou à créer. Les actions restrictives et correctives pourraient facilement voir le jour par le biais de plans d'urbanisme municipaux. Enfin, les actions de développement culturel ponctuel et régional font appel aux ressources gouvernementales, mais devraient être effectuées prioritairement selon les désirs de la population de la région.

Le développement culturel de Charlevoix pourrait s'effectuer à partir de plusieurs pôles, chacun ayant une vocation spécifique. Ces pôles devraient être reliés à l'intérieur d'un circuit de découverte culturelle ayant pour fonction d'animer simultanément toutes les parties du territoire et pas seulement quelques points privilégiés. De cette manière, le développement culturel de Charlevoix, confié en grande partie à la population locale, avec un appui technique et financier adéquat des ministères concernés, pourrait devenir un processus dynamique et original, qui n'entraverait pas pour autant les autres formes de développement économique de la région.

\section{CONCLUSION}

Par l'analyse morphologique nous avons tenté non seulement de définir les caractéristiques des paysages de Charlevoix mais encore de leur attribuer une "valeur " en fonction de critères pertinents à la notion d'arrondissement naturel ou "culturel », telle que définie dans la Loi 2. La méthode utilisée 
répond-t-elle au problème posé ? Pour répondre à cette question il faudrait connaître les modalités d'application de la Loi 2, ou mieux, les éléments d'une politique globale de protection et de mise en valeur des paysages. Par ailleurs, le problème de la protection et de la mise en valeur des paysages ne peut être dissocié du problème plus général de l'aménagement du territoire. Rien ne sert d'isoler et de protéger des portions de territoires aux paysages remarquables si les environs sont soumis à un développement anarchique. En d'autres mots, la sauvegarde des paysages est liée à l'élaboration d'une politique globale de l'aménagement du territoire. La mise en valeur des paysages, quant à elle, devrait être liée à une politique globale de développement culturel et pas seulement de développement touristique. Les paysages, considérés comme un produit culturel, appartiennent d'abord au patrimoine culturel d'une nation et ne doivent pas être considérés uniquement comme une marchandise touristique.

Dans le contexte socio-économique et socio-politique nord américain, le paysage est une marchandise qui se vend. II en découle que la démarche du géographe, expert en paysages par excellence, n'est pas neutre. Tout travail d'évaluation des paysages risque d'être récupéré à des fins qui n'ont rien à voir avec la sauvegarde du patrimoine culturel national (voir Dion et $\mathrm{De}$ Koninck, 1976). D'où l'urgence d'élaborer une politique d'aménagement du territoire et de développement culturel qui assure la protection des paysages au profit de l'ensemble de la société plutôt que de servir les intérêts économiques d'une minorité. Dans ce sens le rôle du géographe n'est pas seulement technocratique, il est ausi politique.

\section{NOTES}

1 Le groupe PAISAGE (Projet d'Analyse et d'Inventaire des Sites et Arrondissements Géographiques) a travaillé sur ce projet, subventionné par le ministère des Affaires culturelles, de septembre 1974 à mars 1976. Les trois responsables, professeurs au département de géographie de l'université Laval, étaient Luc Bureau, Jean Raveneau et Marcel Bélanger. Trois professeurs du département d'histoire ont participé activement au projet: Jean Du Berger (ethnographe), Luc Noppen et Raymond Vézina (historiens de l'art). Des experts en anthropologie, climatologie, écologie, géomorphologie et histoire ont également été consultés. Quinze assistants de recherche ont collaboré aux diverses phases du projet. Le raport final consiste en 8 volumes (dont un atlas) déposés en mars 1976 à la Direction générale du patrimoine du ministère des Affaires culturelles du Québec; le rapport était encore inédit au moment de la publication de cet article.

Le contenu de cet article est étroitement inspiré de discussions et travaux menés à l'intérieur du groupe PAISAGE par les assistants de recherche et les collègues cités plus haut. Nous remercions en particulier Jacqueline Beaulieu et Michel Lecarpentier qui ont collaboré de manière très active aux recherches de base sous la direction de l'auteur.

2 Le Projet de loi 2 a été sanctionné le 8 juillet 1972 par l'Assemblée nationale du Québec (troisième session, vingt-neuvième législature).

3 Assemblée nationale du Québec, Projet de loi 2 ( 8 juillet 1972), article 45. Les italiques sont de nous.

4 Voir les articles de Luc Bureau, Raymond Vézina.

5 Pour cette revue de quelques travaux déjà publiés nous avons consulté, entre autres, deux intéressants documents de synthèse : l'ouvrage de Coomber et Biswas (1973), l'article de Matley (1977), cités en bibliographie

6 Il s'agit ici du territoire couvert par les divisions de recensement de Charlevoix-Est et Charlevoix-Ouest et non de la circonscription électorale provinciale de Charlevoix, plus étendue à l'ouest. 
7 Voir l'article de Jean Vézina dans ce même riuméro.

8 Pour les relevés de terrain, la prise de photographies et l'analyse visuelle en général, nous avons bénéficié de l'assistance précieuse de Michel Lecarpentier.

9 Classification établie par Michel Lecarpentier, sous la direction de l'auteur.

10 Les calculs sur ordinateur ont été effectués par $M$. Clément Nolet que nous remercions ici.

11 Enquêtes effectuées sous la direction de Luc Bureau. Voir l'article de cet auteur.

12 Les idées exprimées dans les lignes qui suivent ont en grande partie été élaborées lors de discussions communes avec nos collègues Luc Bureau, Jean Du Berger et Luc Noppen, dans le cadre du groupe PAISAGE.

\section{BIBLIOGRAPHIE}

L'analyse des paysages, Numéro spécial de l'Espace géographique, Paris, vol, 2 (3), 1973, Voir également, dans la mème revue, le vol. 3 (2), 1974, consacré au thème Paysages et sémiologie.

APPLETON, Jay (1975) The Experience of Landscape. London, Wiley, 293p.

BERTIN, Jacques (1974) Sémiologie graphique. Paris, Mouton et Gauthier-Villars. 2 e édition.

CANADA, Ministère de I'Expansion économique régionale (1969) Potentiel des terres à des fins récréatives Ottawa, Information Canada, 1970. No de cat. RE 63-6/1969F. Rapport No 6, 95p.

COOMBER, Nicholas H. and BISWAS, Asitk (1973) Evolution of Environmental Intangibles. Brorixville, Genera Press, P.O. Box 336, Bronxville, N.Y. 10708. 77p. Voir notamment le chap. 3 , p. 33-49.

DEARINGER, John A. (1968) Esthetic and Recreational Potential of Small Naturalistic Streams near Urban Areas. Lexington, Kentucky, Water Resources Research Institute, University of Kentucky, 260p. Research Report No 13.

DION, Marc et DE KONINK, Rodolphe (1976) L'État et l'aménagement: Orléans, une île à vendre. Cahiers de géographie de Québec, 20 (49) : 39-68.

FINES, K.D. (1968) Landscape Evaluation: A Research Project in East Sussex. Regional Studies, $2: 41-55$

GILG, Andrew W. (1976) Assessing Scenery as a Natural Resource: Causes of Variation in Linton's Method. Scottish Geographical Magazine, 92 (1): 41.49.

JURDANT, M. et al (1972) Carte écologique de la région Saguenay-Lac-Saint-Jean. Québec, Environnement Canada, Centre de recherche forestière des Laurentides. 93 pages, annexes et cartes. Voir p. 62-67.

JURDANT, M., BÉLAIR, J.L., GÉRARDIN, V. et DUCRUC, J.P. (1977) L'inventaire du Capital-Nature. Québec, Pêches et Environnement Canada, 202p. Distribué par Approvisionnements et Services Canada, Ottawa, K1A OS9.

Landscape Evaluation. Manchester, University of Manchester, Centre for Urban and Regional Research, 1976, 304p. Report of the Lanscape Evaluation Research Project.

LAVIGNE, Jacques (1971) Les facteurs géographiques de l'utilisation des eaux du bassin de la rivière Malbaie. Québec, Université Laval, Département de géographie. 115p. Mémoire de recherche de 1 er cycle, non publié.

LEOPOLD, Luna B. and MARCHAND, M.O. (1968) On the Quantitative Inventory of the Riverscape. Water Resource Research, 4 (4).

LEWIS, Pierce F., LOWENTHAL, David and TUAN, Yi-Fu (1973) Visual Blight in America. Washington, D. C. Association of American Geographers, Commission on College Geography. 48p. Resource Paper No 23.

LINTON, D.L. (1968) The Assessment of Scenery as a Natural Resource. Scottish Geographical Magazine, $84: 219-238$

LONG, Gilbert (1969) Conceptions générales sur la cartographie biogéographique intégrée de la végétation et de son écologie. Annales de géographie, (427) 257-285.

LONG, Gilbert (1974-1975) Diagnostic phyto-écologique et aménagement du territoire. Paris, Masson, 2 vols, 256 et $232 \mathrm{p}$. Collection d'écologie, 4 et 5 .

LOWENTHAL, David and Hugh C. PRINCE (1966) The English Landscape. Geographical Review, 54 (3): $309-346$.

LOWENTHAL, David (1965) English Landscape Tastes. Geographical Review, 55 (2): 186-222.

LOWENTHAL, David (1968) American Scene. Geographical Review, 58 (1): 61-88

LYNCH, Kewin (1975) L'image de la ville. Paris, Dunod. 222p.

MATLEY, Ian M. (1977) The Evaluation and Preservation of the Cultural Landscape, in WINTERS, H.A. and WINTERS, M.K. (1977) Applications of Geographic Research. East Lansing, Michigan State University. Department of Geography, p. 61-73.

MCHARG, Ian L. (1969) Design with Nature. New York, The Natural History Press, 197p.

MONTREAL (ville) Service d'urbanisme. Relevé visuel, région de Montréal. Première partie, Relevé d'ensemble, 1966. 82p., 17 cartes. Deuxième partie, I'archipel d'Hochelaga, Bulletin technique No 6, sept. 1971, 51 p. 
QUÉBEC, (Prov.) Ministère des Terres et Forêts, Service de l'Aménagement des terres (1974) Inventaire écologique de lile d'Anticosti. Voir la section sur "L'évaluation de la récréation et des paysages ", rédigée par $P$. Watson (Gauthier, Poulin, Thériault et associés).

RONDOT, Jehan (1970) La structure de Charlevoix comparée à d'autres impacts météoritiques. Canadian Journal of Earth Sciences, VII: 1194-1202.

La science du paysage. Numéro spécial de la Revue géographique des Pyrénées et du Sud-Ouest, 43 (2), 1972.

SHAFER, E.L., Hamilton, J.F. and SCHMIDT, E.A. (1969) Natural Landscape Preferences : A Predictive Model. Journal of Leisure Research, 1 (1).

SHAFER, E.L. and MIETZ, J. (1970) It Seems Possible to Quantity Scenic Beauty in Photographs. Upper Darby, Pennsylvania, Northeastern Forest Experiment Station, U.S.D.A. Forest Service Research Paper, NE-162.

\section{RÉSUMÉ}

RAVENEAU, Jean : Analyse morphologique, classification et protection des paysages: le cas de Charlevoix.

L'objectif de cette recherche est de trouver des méthodes d'analyse et de classification des paysages devant permettre l'application des articles de la Loi sur les biens culturels (Loi 2, 1972, Province de Québec) concernant la déclaration "d'arrondissements naturels». La méthodologie proposée a été appliquée à la région de Charlevoix située à une centaine de $\mathrm{km}$ au nord-est de la ville de Québec.

On montre d'abord que les "arrondissements naturels" décrits dans la loi devraient plutôt porter le nom "d'arrondissements culturels ". L'identification des portions de territoire pouvant être déclarés arrondissements naturels ou culturels implique une analyse préalable des paysages de l'ensemble d'une région. Trois dimensions doivent être considérées : les formes. le peuplement, la perception. Dans cet article on aborde uniquement le problème de l'étude des formes des paysages, c'est-à-dire l'analyse morphologique. Quelques méthodes modernes d'analyse des paysages sont passées en revue. Elles comportent généralement trois phases: inventaire, classification et évaluation proprement dite en fonction de critères particuliers (récréation notamment).

La région de Charlevoix se prête particulièrement bien à l'étude des paysages en raison de sa topographie contrastée, de l'ancienneté de son peuplement, de l'empreinte d'activités économiques diversifiées. On a d'abord procédé à une analyse visuelle comportant le relevé des limites visuelles, la classification des points de vue et le relevé des types d'itinéraires routiers. On a ensuite dressé un inventaire spatial et monté une matrice géographique d'informations comportant 70 variables relevées sur la base de 47 zones de travail. On a choisi des variables qui structurent l'image des paysages et qui en conditionnent l'évolution, à la fois dans l'espace et dans le temps. La matrice a été traitée simultanément selon des méthodes visuelles (méthode Bertin) et quantitatives (analyse factorielle). Ce traitement a fait ressortir l'influence structurante du relief, de l'ancienneté et de la densité du peuplement, de l'importance du patrimoine historique. Quatre grands ensembles géographiques ont ainsi été dégagés, chacun comportant plusieurs sous-ensembles: les zones montagneuses sans population, les zones de peuplement récent du plateau. les vallées et leurs rebords, le littoral. Une seconde classification porte spécifiquement sur les variables pouvant permettre de définir le "potentiel culturel ". Elle conduit à une hiérarchisation des zones de Charlevoix en fonction de leur intérêt plus ou moins grand pour une désignation éventuelle comme arrondissement "naturel" (ou culturel). Cette classification est complétée par l'identification des diverses formes de pollution des paysages. La principale menace de dégradation des paysages provient des poussées d'urbanisation anarchique à la périphérie de Baie-Saint-Paul et La Malbaie.

En conclusion il apparaît que la notion même d'arrondissement culturel ne permet pas de répondre aux impératifs de protection et de mise en valeur des paysages. II faut plutôt penser en termes de développement culturel global impliquant des actions d'intensité différente selon les types de paysages et les dangers de dégradation les affectant.

MOTS-CLÉS: Analyse et classification des paysages, arrondissements culturels, analyse visuelle, matrice géographique d'informations, pollution visuelle. Région de Charlevoix, Province de Québec. 


\section{ABSTRACT}

RAVENEAU, Jean: Morphological analysis, classification and protection of landscapes : the case of Charlevoix.

The aim of this research is to find methods of landscape analysis and classification which would allow the enforcement of sections of the Cultural Property Act (Bill 2, 1972, Province of Québec) specifically those dealing with the declaration of «natural districts ». The proposed methodology was applied to the Charlevoix region, located one hundred km north-east of Québec City.

First the author demonstrates the misnaming of «natural districts» which should be called «cultural districts». The identification of parts of territory that may be declared natural or cultural districts involve a previous landscape analysis of the whole region. Three components of landscape should be considered: shapes, settlement and perception. This paper deals only with the problem of shapes study, namely morphological analysis. Some modern methods of landscape analysis are reviewed. Three phases are generally involved: inventory, classification and evaluation depending on particular assumptions (i.e.: recreation).

The Charlevoix region is well fitted for a landscape study because of its strong topography, its early settlement and the mark of various economic activities on its landscape. First, a visual analysis was made, including a survey of visual boundaries, classification of vistas and a survey of road itinerary patterns. The next step was to make a spatial inventory and to build a geographical data matrix comprising 70 variables pertaining to 47 working zones. Variables which frame the landscapes image and evolution in space and time were selected. The data matrix was handled both with visual and quantitative methods (Bertin's method and factor analysis). The leading factors revealed by data handling are the relief, the age and density of settlement and the importance of historical patrimony. Four major geographical areas were found, each comprising several sub-areas: mountainous unpopulated areas, late settlement plateau areas, the valleys and their edges and the St. Lawrence coast-line. A second classification involves only the variables tied with the definition of «cultural potential». The result is a grading of Charlevoix areas according to their potential as a possible choice for natural or cultural districts. This classification is suplemented by the identification of various kinds of landscape pollutions. The main threat of landscape damage comes from the disordered spreading of townscapes around Baie-Saint-Paul and La Malbaie (Murray Bayl.

A concluding remark is that the concept of cultural district does not offer a satisfying answer to the requirements of landscapes protection and development. One must rather think of a global cultural development involving various actions graduated according to landscape patterns and threats to harming them.

KEY WORDS: Landscape analysis and classification, cultural districts, visual analysis, geographical data matrix, visual pollution. Charlevoix region, Province of Québec. 